OPEN ACCESS

Edited by:

Jiexi Wang,

Central South University, China

Reviewed by:

Lifang Jiao,

Nankai University, China

Wei Luo,

Tongji University, China

${ }^{*}$ Correspondence:

Qinghao L

lqh@iphy.ac.cn

Xiqian Yu

xyu@iphy.ac.cn

Specialty section:

This article was submitted to

Physical Chemistry and Chemical

Physics,

a section of the journal

Frontiers in Chemistry

Received: 31 August 2018 Accepted: 29 November 2018 Published: 12 December 2018

Citation:

Nie K, Hong Y, Qiu J, Li Q, YU X, Li H and Chen L (2018) Interfaces Between Cathode and Electrolyte in Solid State Lithium Batteries: Challenges and Perspectives. Front. Chem. 6:616. doi: 10.3389/fchem.2018.00616

\section{Interfaces Between Cathode and Electrolyte in Solid State Lithium Batteries: Challenges and Perspectives}

\author{
Kaihui Nie ${ }^{1,2}$, Yanshuai Hong ${ }^{1,2}$, Jiliang Qiu ${ }^{1,2}$, Qinghao $\mathrm{Li}^{1,2 *}$, Xiqian $\mathrm{Yu}^{1,2 *}$, Hong $\mathrm{Li}^{1,2}$ and \\ Liquan Chen ${ }^{1,2}$
}

${ }^{1}$ Renewable Energy Laboratory, Institute of Physics, Chinese Academy of Sciences, Beijing, China, ${ }^{2}$ University of Chinese Academy of Sciences, Beijing, China

Solid state lithium batteries are widely accepted as promising candidates for next generation of various energy storage devices with the probability to realize improved energy density and superior safety performances. However, the interface between electrode and solid electrolyte remain a key issue that hinders practical development of solid state lithium batteries. In this review, we specifically focus on the interface between solid electrolytes and prevailing cathodes. The basic principles of interface layer formation are summarized and three kinds of interface layers can be categorized. For typical solid state lithium batteries, a most common and daunting challenge is to achieve and sustain intimate solid-solid contact. Meanwhile, different specific issues occur on various types of solid electrolytes, depending on the intrinsic properties of adjacent solid components. Our discussion mostly involves following electrolytes, including solid polymer electrolyte, inorganic solid oxide and sulfide electrolytes as well as composite electrolytes. The effective strategies to overcome the interface instabilities are also summarized. In order to clarify interfacial behaviors fundamentally, advanced characterization techniques with time, and atomic-scale resolution are required to gain more insights from different perspectives. And recent progresses achieved from advanced characterization are also reviewed here. We highlight that the cooperative characterization of diverse advanced characterization techniques is necessary to gain the final clarification of interface behavior, and stress that the combination of diverse interfacial modification strategies is required to build up decent cathode-electrolyte interface for superior solid state lithium batteries.

Keywords: cathode, solid electrolyte, solid state lithium battery, cathode-solid electrolyte interface, advanced characterization

\section{INTRODUCTION}

The daily increasing energy consumption demands advanced batteries with higher energy density and superior safety performance, particularly for large-scale applications like electric vehicles and grid storage (Tarascon and Armand, 2001). In solid state lithium batteries, conventional liquid electrolyte based on flammable carbonate components is replaced by solid electrolyte. Thereby, the safety concern related to thermal runaway and electrolyte combustion is likely to be much 
mitigated (Zhang et al., 2013). Owing to the mechanical properties of solid electrolyte, solid state lithium batteries could resist lithium dendrite in a great degree and the cycle life could be extended longer than lithium batteries based on liquid electrolyte. The wide electrochemical stability window of solid electrolyte may further enable the application of Li metal as anode and cathodes with even higher oxidization potential. With larger lithium chemical potential difference between anode and cathode, the energy storage can be much improved correspondingly. Owing to these glittering properties, solid state lithium batteries have attracted much research attention in recent years and become promising candidates for next generation energy storage devices with the expectations of improved safety performance, longer cycle life, and higher energy density (Bates et al., 2000; Duan et al., 2017). Depending on whether the battery contains liquid electrolyte or not, solid state lithium batteries can be divided into all solid state lithium batteries and hybrid solid liquid electrolyte lithium batteries (Cao et al., 2018).

The development of practically accessible solid state lithium batteries is hindered by two major bottle-necks. The first one is the low ionic conductivity of solid electrolyte, which is severalorders-lower than that of liquid electrolyte at room temperature (RT). Continuous research efforts have been devoted to designing superior solid electrolyte in the past decades, and much progress has been achieved so far. Up to now, the RT ionic conductivities of some systems have approached or even surpassed that of liquid electrolytes. RT conductivities of NASICON-type oxides (Aono et al., 1990; Fergus, 2012) and lithium garnets (Murugan et al., 2007) have reached $\sim 1 \mathrm{mS} \mathrm{cm}^{-1}$. Kato et al. further increased the number to $\sim 25 \mathrm{mS} \mathrm{cm}{ }^{-1}$ in $\mathrm{Li}_{9.54} \mathrm{Si}_{1.74} \mathrm{P}_{1.44} \mathrm{~S}_{11.7} \mathrm{Cl}_{0.3}$ (Kato et al., 2016). Decent solid electrolytes are now available with intrinsically high $\mathrm{Li}^{+}$conductivity, Lithium ion transference number $t_{\mathrm{Li}+} \sim 1$, and particularly no desolvation step compared to organic liquid electrolytes, $\mathrm{t}_{\mathrm{Li}+}$ is around $0.20 .5(\mathrm{Xu}$, 2004; Zugmann et al., 2011). Corresponding solid state lithium batteries are expected to exhibit large capacities and high power densities for future applications (Kato et al., 2016).

Despite the rapid development of solid electrolyte itself, the even more serious hinderance for solid state lithium batteries is the high interfacial resistance caused by poor contact and interfacial reactions (Zhang et al., 2016). Without liquid fluidity, it's challenging to obtain intimate contact between solid electrolyte and electrode. The periodic electrode expanding and shrinking during cycle further deteriorates the mechanical particle-to-particle contact. As a consequence, high polarization, and low utilization of active materials are conventional in solid state lithium batteries. Meanwhile, the high voltage instability of solid electrolyte is another noteworthy concern for solid state lithium batteries. Solid electrolytes are expected to provide wider electrochemical stability window compared with liquid electrolyte. Literatures also reported wide electrochemical window up to $5.0 \mathrm{~V}$ or even $6.0 \mathrm{~V}$ in inert electrode system. However, some computation results and experimental results confirmed that the window is not as high as reported before (Han et al., 2016). Especially, for SPE the prevailing experimental reports of which are mostly based on $\mathrm{LiFePO}_{4}$ cathode cycled within $3.8 \mathrm{~V}$. Considering the catalytic behavior of transition metal oxides, the practical stability of solid electrolyte at high voltage still needs further investigation, and verification. Moreover, the electric potential profile across the electrodeelectrolyte interface is still a problem unanswered, which has significant influences on interface reaction and battery performance. Many investigations have been carried out on the abrupt change of electric potential across cathode-electrolyte interface (Liang et al., 2018). And it has been pointed out that the interfacial side reactions may be accelerated dramatically due to the specific local electric potential. However, an intensive and systematic understanding is still lacking on the potential profile distribution across the interface and its corresponding influence on the interface behavior.

It can be inferred from above that the key to realize solid state lithium batteries with competitive performance mostly relies on the construction of a stable and intimate interface, where different strategies have been developed. Direct co-sintering of electrode and electrolyte may be an effective and simple method to achieve good interfacial contact. However, the high temperature facilitates ion interdiffusion across interface, leading to side reactions between the electrode and solid electrolyte. Insitu synthesis of solid electrolyte or cathode is another promising choice, but necessary sintering procedure also encounters the problem of ion interdiffusion. Due to the interfacial passivation layer formation, the dynamics performance of solid state lithium batteries may be deteriorated. Diverse strategies have been proposed to build up proper artificial interlayer, including cathode coating, interface softening, buffer layer introducing, and etc. These strategies can effectively improve the physical contact, diminish interfacial side reactions, and mitigate the space charge layer (SCL) in sulfide solid electrolyte, but corresponding solid state lithium batteries are still far from practical applications. Till now much efforts have been devoted to interface modification and progresses have been obtained, but interface property is still a major obstacle on the way to practical solid state lithium batteries.

Interface research has become a challenging but hot topic in solid state batteries (Gao et al., 2018) (Lu et al., 2018; $\mathrm{Xu}$ et al., 2018). The interface between lithium anode and solid electrolyte has been extensively investigated. Note that the cathode-solid electrolyte interface serves as a hinge to obtain batteries with improved safety, longer cycle life, and higher energy density. So, the interface between cathode and solid electrolyte is equally important to the interface at anode side. Here in this review, we put a special focus on the fundamental issues about cathode-solid electrolyte interfaces in solid state lithium batteries based on diverse cathode-electrolyte materials. We hope to summarize the previous understandings and recent advances on the interface research. Furthermore, we hope to shed light on the possible approach to the final understanding of interface phenomenon with advanced characterization techniques. In chapter 2, we present a brief overview on basic principle of battery operation and scientific issues relevant to interface layer. In chapter 3, the interfacial problems between cathodes and four kinds of prevailing solid electrolytes are specifically discussed, corresponding optimization methods are also introduced. In chapter 4, advanced characterization techniques used for the 
investigation of solid-solid interface behavior are consolidated, corresponding advances and achievements are summarized. Finally, we give a comprehensive conclusion about the cathodesolid electrolyte issues and perspectives for building favorable interfaces.

\section{BASIC PRINCIPLE AND ISSUES AT THE CATHODE-SOLID ELECTROLYTE INTERFACE}

Solid state lithium batteries have three major components cathode, anode, and solid electrolyte. The cathode material herein refers to the same lithium-containing compound as the lithium ion battery. During charging, $\mathrm{Li}^{+}$are extracted from the cathode and migrate to anode via solid electrolyte, while electrons transfer from the cathode to anode through external circuit. In this process, oxidation and reduction reactions take place at the cathode and anode sides, respectively. During discharging, $\mathrm{Li}^{+}$and electrons migrate toward the reverse direction, accompanied with cathode reduction, and anode oxidation. The following reaction steps are involved at electrodeelectrolyte interface in solid state lithium batteries: (i) $\mathrm{Li}^{+}$ diffusion in the electrolyte, (ii) $\mathrm{Li}^{+}$hop into the first lattice site of the electrode and oxidation/reduction reaction happened at the same time i.e., the charge transfer process, (iii) $\mathrm{Li}^{+}$diffusion in the electrode, and (iv) Surface reaction, etc. A stable and intimate interface is necessary to ensure the above reaction steps proceed smoothly.

Interface instability may derive from chemical or electrochemical problems, a most fundamental origin is the abrupt electrochemical potential change at electrodeelectrolyte interface. As illustrated in Figure 1A, the lowest unoccupied molecular orbital (LUMO) and highest occupied molecular orbital (HOMO) of electrolyte determines the electrochemical stability window of solid state lithium batteries. The electrochemical potential of anode and cathode is marked as $\mu_{\mathrm{A}}$ and $\mu_{\mathrm{C}}$, which need to match with the electrochemical window of electrolyte to achieve thermodynamic stability (Goodenough, 2013). Figure 1B shows the ionic and electronic structures of electrode and electrolyte before and after contact, where electrolyte exhibits higher $\mathrm{Li}^{+}$chemical potential. $\mathrm{Li}^{+}$ will migrate from solid electrolyte to oxide cathode to achieve thermodynamic equilibrium. This will lead to the alignment of $\mathrm{Li}^{+}$electrochemical potentials and a space charge layer (SCL) formation with an inner electric field after contacted. While band bending and alignment of Fermi level will happen due to the formation of a heterojunction. As a result, original position of energy levels, inner electric field formation, band bending as well as the energy levels change during charging/discharging determine barriers for charge carriers transfer. From the barriers for charge carriers transfer, whether electrons/holes could transfer at the interface i.e., the oxidation/reduction of the electrolyte, could be concluded. (Hausbrand et al., 2014) It was pointed out that side reactions at interface may be further accelerated due to the large polarization from electric potential $(\varphi)$ drop (Ohta et al., 2006; Zhou et al., 2016). In such cases, solid electrolyte decomposition and intermediate transition layer formation may take place at interface. In addition, conventional high temperature processing may further induce interfacial interdiffusion of TM (transition metal) elements and favor the formation of specific transition region.

Based on the intrinsic properties of different kinds of solid electrolytes and cathode materials, there are mostly three types of electrode-electrolyte interfaces in solid state lithium batteries, as shown in Figure 1C (Zhu et al., 2016). Type 1 is a stable interface scenario with no electrolyte decomposition or chemical side reactions. This is the ideal interface, which seldomly appears in practical systems. Type 2 represents interface which is electronic insulating but provides $\mathrm{Li}^{+}$migration channels. Within this scheme, further interfacial side reactions can be suppressed and battery operation can be maintained. The $\mathrm{LiCoO}_{2} / \mathrm{LiPON}$ interface may be a proper example for this case ( $\mathrm{Zhu}$ et al., 2016). Type 3 is an undesirable but most common interface with mixed ionic and electronic conductivity. In this scheme, continuous side reactions occur, and battery fade happens, as in $\mathrm{LiCoO}_{2} / \mathrm{LGPS}$ interface. Depending on the intrinsic property of electrode and electrolyte, different types of interfaces will be built up, but only type 1 and 2 are accessible for practical applications. By introducing proper buffer layers between cathode and electrolyte, a stable artificial layer can be constructed and convert interface from type 3 to type 2 . Considering the significance of building proper $\mathrm{Li}^{+}$conducting layer and balancing interfacial potential drop, we will present detailed discussion in following chapters according to the characteristics of specific solid electrolytes.

Apart from the chemical stability of the interface, mechanical behavior also has a significant impact on battery performance. In conventional lithium ion batteries based on liquid electrolyte, cathode particles can be totally immersed in liquid electrolyte and passivation layer called solid electrolyte interphase (SEI) may form. Good contact between electrode and liquid electrolyte could therefore be maintained throughout battery cycle, Figure 2A (Liu et al., 2006; Takamatsu et al., 2012). However, it is challenging to maintain intimate electrode-electrolyte interface in solid state lithium batteries, especially over many cycles (Goodenough, 2013). The deficient contact in solid state lithium batteries may well-lead to low utilization of active particles, large polarization and even contact loss during cycle.

Due to the distinguished mechanical properties, there is distinct difference in contact behavior among various types of electrolytes. Solid electrolytes can be generally classified into SPE and solid inorganic electrolyte, the latter can be further classified into solid oxide and solid sulfide electrolyte. Polymer electrolyte has moderate contact with cathode due to the elasticity and deformability of organic polymers. Nevertheless, vacant cavities will still generate due to interface reactions and cathode pulverization during cycling (Figure 2B) (Nakayama et al., 2010). The effective contact area between cathode and polymer electrolyte will consequently reduce with battery cycle. Due to reasonable mechanical ductility, deformable sulfide particles could also change its shape to match with cathode particles. Hence, the poor contact between electrode and sulfide electrolyte can be much improved by mechanical pressing 


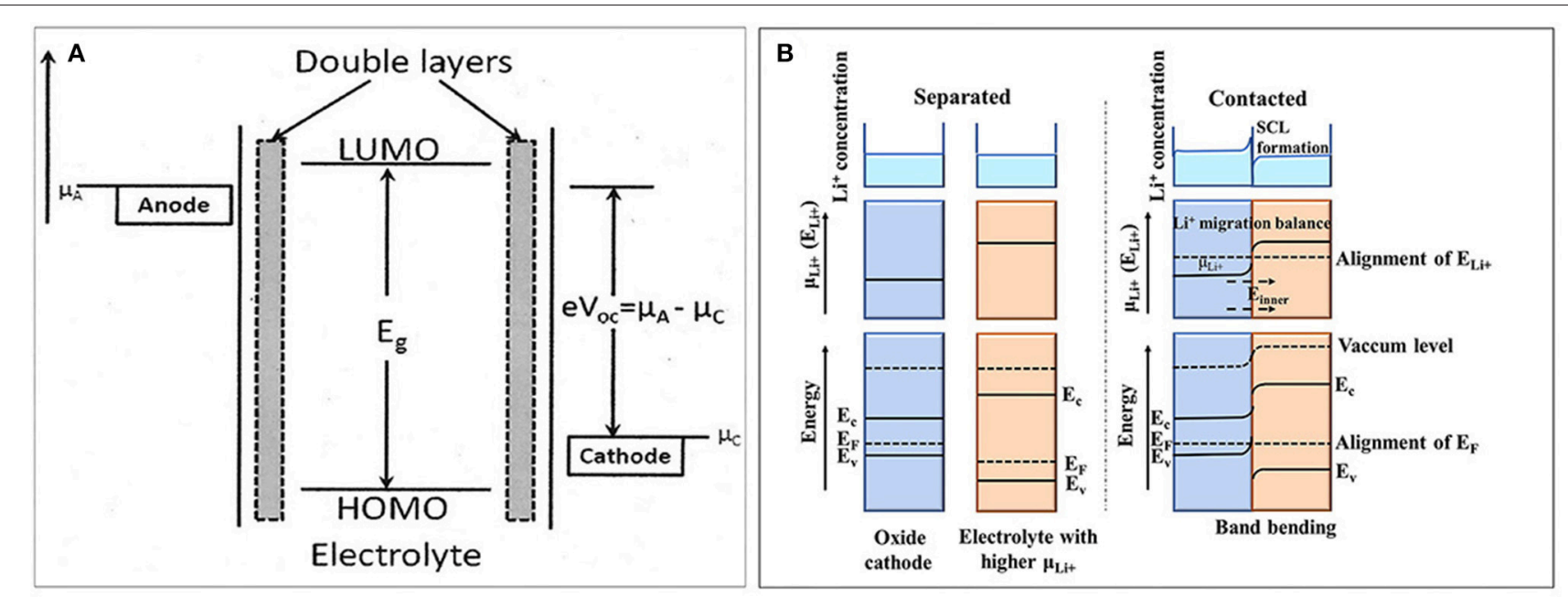

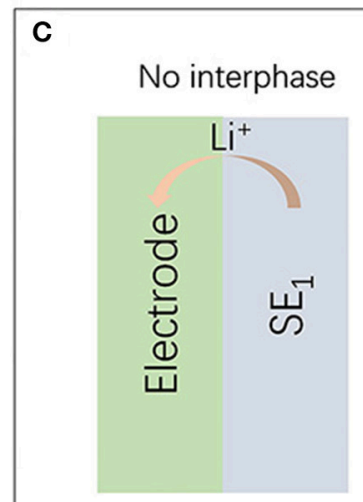

Type 1.

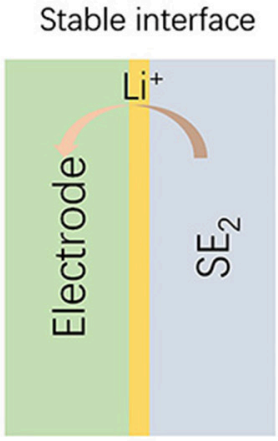

Type 2.

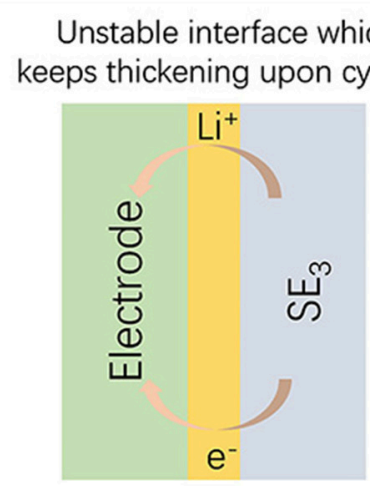

Type 3.

FIGURE 1 | (A) Relative energies of $\mu_{A}$ and $\mu_{C}$ vs. the LUMO-HOMO window of the electrolyte. [Reprinted with permission from Goodenough (2013). Copyright (2013) American Chemical Society]. (B) Illustration of ionic and electronic structures of electrode and electrolyte before (left) and after contact (right). Shown is a mixed ionic and electronic conducting electrode in contact with a pure ionic conducting solid electrolyte with higher $\mathrm{Li}^{+}$chemical potential. (C) Illustration of three possible types of the solid electrolyte/solid electrode interfaces.

(Figure 2C) (Sakuda et al., 2013; Ito et al., 2017). While contact loss will also happen upon cycling along with the shrinkage and expansion of cathode particles (Koerver et al., 2017). Solid oxide electrolytes have the worst point-contact with cathode due to the rigid ceramic nature (Figure 2D) (Ohta et al., 2013, 2014). The insufficient mechanical contact facilitates cathode particles completely isolated from solid electrolyte, i e., the "dead" area. Due to the lack of percolation paths, neither electrons nor $\mathrm{Li}^{+}$can be transferred from/into the dead areas. The "dead" areas not only lead to direct capacity fading, but also induce locally strong non-uniform current and strain distribution (Zhang et al., 2018). The poor solid-solid contact typically brings about large polarization and low capacity. To improve the interface contact, various strategies have been adopted, such as in-situ synthesis of solid electrolyte, interface buffer layer, cathode coating, gel system etc. Based on different properties of various electrolytes, specific strategies will be adopted, and introduced specifically in Chapter 3.

\section{CHALLENGES AND SOLUTIONS ON INTERFACES BETWEEN CATHODE AND DIVERSE SOLID ELECTROLYTES}

\section{Interface Between Cathode and Solid Polymer Electrolyte}

After Wright's discovery of alkali metal ions conductivity in poly(ethylene oxide) (PEO) in 1973 (Fenton et al., 1973), Armand firstly proposed PEO with lithium salt as solid electrolyte for solid state lithium batteries (Armand, 1983). PEO-based SPE is widely accepted as a most promising candidate for solid state lithium batteries owning to its advantages such as easy fabrication, low cost and excellent compatibility with lithium salt. In SPE, $\mathrm{Li}^{+}$can migrate in the free volume of polymer host assisted by the motion of the polymer chains when temperature is above $\mathrm{T}_{\mathrm{g}}$ (glass transition temperature) (Bruce, 1995). In PEO based SPE, $\mathrm{Li}^{+}$were coordinated by ether oxygen and transport with the breaking/forming of Li-O bonds (Bruce, 1995; Xu, 2004). However, PEO-based SPE is not stable above 


\section{A Liquid electrolyte}

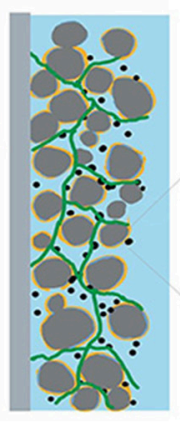

B Solid polymer electrolyte

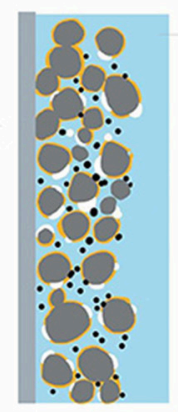

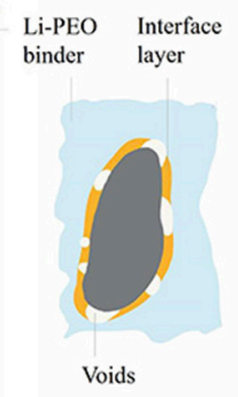

C Solid sulfide electrolyte

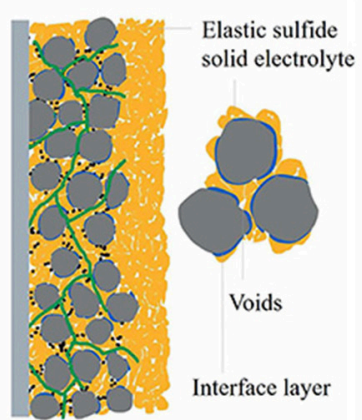

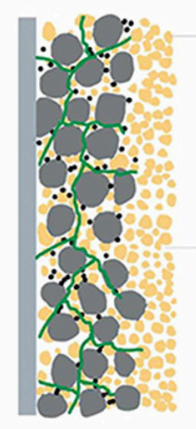

electrolyte

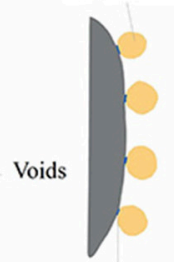

Interface layer

\section{Current collector}

\section{Cathode particle}

\section{$\because$ Conducting additives $\downarrow$ Binder}

FIGURE 2 | Models of morphology at the interface between cathode-electrolyte: (A) The cathode particles are totally immersed in a liquid electrolyte and an interface layer will form. (B) Cathode particles are distributed in a Li-PEO binder with good contact while voids will generate upon cycling because of the interfacial reactions or pulverization of cathode particles. (C) Sulfide particles have favorable mechanical properties as ductility and deformability, which could change its shape to match with the rigid solid electrode. (D) Solid oxide electrolyte: Poor point-contact will form due to the rigid ceramic nature. Interface layer will form in all the aforementioned system if decomposition reactions or interdiffusion occurred at the interface.

$4.0 \mathrm{~V}$, which confines the pairing cathode operating within lowvoltage range. In most literature reports, the prevailing choice is $\mathrm{LiFePO}_{4}$ (Croce et al., 2001). Considering the catalytic effect of transition metal oxides, PEO decomposition may well-be triggered at the interface region. And improving the antioxidant properties of SPE to high-voltage range is essential to realize high energy density solid state lithium batteries based on PEO (Fan et al., 2002). There are mostly three types of SPE available (i) dry solid-state polymer, (ii) gel/plasticized polymer electrolyte, and (iii) polymer composites (Manuel Stephan, 2006). Diverse optimization strategies have been utilized for different SPE systems, as discussed below.

"Dry" solid-state polymer refers to PEO, PPO, PAN etc. and their derivatives containing Li salt, corresponding optimization has been focused on polymer architecture and Li salt selection. On the electrochemical instability of PEO-based SPE above $4.0 \mathrm{~V}$ (Croce et al., 2001), Nakayama et al. (2010) proposed a model that two sequential factors affect cyclic degradation. The first one is the local current enhancement induced by cathode pulverization, which can be attributed to the solid-solid contact between electrolyte and electrode. The second is continuous and uneven decomposition of TFSI $\left[\mathrm{N}\left(\mathrm{CF}_{3} \mathrm{SO}_{2}\right)_{2}^{-}\right]$due to local polarization. These results indicate that the mechanical property of SPE and the Li salt selection are both essential. Ma et al. proposed a novel SPE composed of PEO and extra-stable lithium salt- $\left(\mathrm{Li}\left[\left(\mathrm{CF}_{3} \mathrm{SO}_{2}\right)\left(\mathrm{n}-\mathrm{C}_{4} \mathrm{~F}_{9} \mathrm{SO}_{2}\right)-\mathrm{N}\right]\right.$, LiTNFSI). This SPE exhibits a homogeneous and compact morphology and high electrochemical stability at $\sim 4.0 \mathrm{~V}$ vs. $\mathrm{Li}^{+} / \mathrm{Li}$ (Figure 3A). Longterm cycling stability and sufficient thermal stability $\left(>350^{\circ} \mathrm{C}\right)$ was also obtained in this novel SPE (Ma et al., 2016b).

Improving the antioxidative capability of PEO is another critical aspect to promote high voltage interface stability. Copolymerization, branching, and crosslinking are common polymer modification methods, which also favor designing more antioxidative polymers (Tong et al., 2014; Porcarelli et al., 2016; Wang et al., 2016a). UV-induced (co)polymerization can promote effective interlinking between polyethylene oxide (PEO) chains plasticized by tetraglyme. Hereby, Porcarelli et al. (2016) obtained SPE with wide electrochemical stability window $(>5 \mathrm{~V}$ vs. $\mathrm{Li} / \mathrm{Li}^{+}$) from LSV test. Figure 3B illustrates the synthesis process and photograph of as-prepared SPE, together with cross-sectional FESEM images of optimized cathode-electrolyte interface. It is clear that electrolyte creates conformal coating by following the contours of active particles, which leads to improved active materials utilization. Similar optimized SPE have also been achieved by a PEO and liquid-crystalline copolymers with small molecular liquid crystals as fillers. High ionic conductivity, lithium ion transference number combined with wide electro-chemical stability window of the copolymer facilitated a good electrochemical performance (Tong et al., 2014).

Except for developing PEO derivatives, exploiting other antioxidative polymer electrolyte has also attracted much attention (Zhang et al., 2015; Chai et al., 2017). Chai et al. (2017) prepared a kind of novel poly (vinylene carbonate) (PVCA)based SPE which possessed both interfacial compatibility with $\mathrm{Li}$ anode and high-voltage $\mathrm{LiCoO}_{2}$ cathode $\left(4.3 \mathrm{~V}\right.$ vs. $\left.\mathrm{Li} / \mathrm{Li}^{+}\right)$. From in-situ polymerization of PVCA, polymer electrolyte can be even incorporated into the porous cathodes and the effective contact area can be much increased as a result. Owing to the good contact and compatibility, the battery exhibited high discharge capacity and excellent cycling performance.

The second category SPE is called "gel polymer electrolyte" or "plasticized polymer electrolyte" which contains both liquid and solid components. Thus, gel polymers possess both cohesive properties of solids and the diffusive property of liquids (Manuel Stephan, 2006) which makes hybrid solid liquid electrolyte lithium batteries have unique advantages (Huang et al., 1996). 
As reported, by modifying PEO electrolyte with plasticizing liquid dimethyl sulphoxide (DMSO) $(<5 \%)$, electrochemical stability window can be extended above $4.1 \mathrm{~V}$, exceeding Fermi levels of several high voltage cathodes. The long terms cycling stability was also improved obviously (Zewde et al., 2018). By phase inversion technique, Deng et al. prepared a microporous polymer electrolyte (MPE) based on poly (vinylidene fluoride) (PVDF)/PEO star polymer, which exhibits wide electrochemical stability window of $\sim 5 \mathrm{~V}$ (Deng et al., 2015).

Solid composite electrolyte, as SPEs subset, is another competitive candidate among kinds of SPEs. Solid composite electrolyte combined the virtues of both polymer and ceramic, exhibiting excellent mechanical stability, high ionic conductivity, wide electrochemical stability window, intimate contact performance, and etc. Relevant researches will be specifically introduced in Chapter 3.4.

Apart from solid electrolyte modification, cathode surface modification is another effective way to mitigate the interface degradation. Note that a principle factor that restricts PEO application at high voltage is the strong oxidation/catalytic property of TM oxides cathode. Consequently, surface modification on cathode material becomes another way to enable PEO operation at high voltage. Yang et al. synthesized continuous and compact LATP coating layer on $\mathrm{LiCoO}_{2}$ through solution-procession and low temperature treatment. solid state lithium batteries assembled with PEO-based SPE and LATP modified $\mathrm{LiCoO}_{2}$ shows high capacity retention $(93.2 \%$ after 50 cycles) at $4.2 \mathrm{~V}$, which suggests that surface coating can effectively suppress PEO oxidation at high voltage (Yang et al., 2018). By cathode coating, PVCA-coated $\mathrm{LiCoO}_{2}$ also showed much enhanced cycling stability of PEO based SPE at 4.45V (Ma et al., 2017).

\section{Interface Between Cathode and Solid Oxide Electrolyte}

Oxide-based solid electrolytes exhibit good chemical stability against air and compatibility with high voltage cathodes. Typical solid oxide electrolytes include garnet-type $\mathrm{Li}_{7} \mathrm{La}_{3} \mathrm{Zr}_{2} \mathrm{O}_{12}$ (LLZO), NASICON-type $\mathrm{LiTi}_{2}\left(\mathrm{PO}_{4}\right)_{3}$, LiSICON-type $\mathrm{Li}_{14} \mathrm{Zn}$ $\left(\mathrm{GeO}_{4}\right)_{4}$, and Perovskite-type $\mathrm{La}_{0.5} \mathrm{Li}_{0.5-\delta} \mathrm{TiO}_{3}$ (LLTO). Solid oxide electrolyte is a most competitive choice for solid state lithium batteries (Chen et al., 1980; Delmas et al., 1988; Inaguma et al., 1993). However, there are two major challenges for solid oxide electrolytes. The first one is the generally low ionic conductivity, which is lower than sulfide electrolytes. Despite the phenomenally low intrinsic bulk conductivity, recent investigations point to the high interface polarization that restrains battery dynamics. The second challenge is the rigid ceramic nature, which causes poor point-contact at electrodeelectrolyte interface, as discussed above. Solid oxide electrolytes have a key advantage of intrinsic wide electrochemical window. For garnet-type electrolytes, the experimental value can be even wide as $\sim 0-6 \mathrm{~V}$ ( $\mathrm{Li}$ et al., 2015; Thangadurai et al., 2015). Among all solid oxide electrolytes, garnet-type electrolyte is an attractive candidate due to its high $\mathrm{RT}$ ionic conductivity $(\sim 1 \mathrm{mS}$ $\mathrm{cm}^{-1}$ ), practically wide electrochemical window and chemical stability against Li etc. In the following discussion, we mostly take garnet LLZO as a typical example to discuss the interfacial problems solid oxide electrolyte faced with, other systems are briefly mentioned at the end.

Since interfacial resistance from poor contact (Figure 2D) is proven to be the main reason for the high internal resistance of solid state lithium batteries (Park et al., 2016; Han et al., 2017), quite a few approaches have been applied to reduce interfacial resistance, including co-sintering (Wakayama et al., 2016), in-situ synthesized electrolyte layer (Yoshima et al., 2016; Kazyak et al., 2017), interface buffer layers (Kato et al., 2014; Park et al., 2016), interface softening (Seino et al., 2011; Sakuda et al., 2012; Liu et al., 2016), surface coating (Han et al., 2017), and amorphous cathode (Matsuyama et al., 2016; Nagao et al., 2017) etc.

Electrode-electrolyte co-sintering, cathode layer in-situ synthesizing, and thin film deposition are proven effective in promoting surface contact, however necessary high-temperature handling $\left(>500^{\circ} \mathrm{C}\right)$ will lead to elements interdiffusion, electrolyte decomposition and deteriorated performance (Wakayama et al., 2016). Tremendous efforts have been devoted to lower the sintering temperature of solid oxide electrolytes to mitigate the interdiffusion problem, while very finite progress has been achieved so far. With a combination of ab initio calculations, thermal analysis, and X-ray-diffraction, Ceder' group elucidated the decomposition reactions between highvoltage spinel cathode $\left(\mathrm{Li}_{2} \mathrm{NiMn}_{3} \mathrm{O}_{8}, \mathrm{Li}_{2} \mathrm{FeMn} \mathrm{O}_{8}, \mathrm{LiCoMnO}_{4}\right)$ and solid oxide electrolyte (LLZO, LATP) (Miara et al., 2016). $\mathrm{XRD}$ revealed that spinel cathode and LLZO were not compatible with each other at $600^{\circ} \mathrm{C}$, and the decomposition products can be predicted from calculated phase diagrams. In 2016, Park et al. (2016) studied the three-dimensional elemental distribution at $\mathrm{LiCoO}_{2} / \mathrm{LLZO}$ interface by TOF-SIMS. As illustrated in Figure 4A, Co diffuses into LLZO, and $\mathrm{Zr} / \mathrm{La}$ diffuses into $\mathrm{LiCoO}_{2}$. While $\mathrm{Al}$ was leached out of LLZO, and diffuses into $\mathrm{LiCoO}_{2}$, cubic LLZO at the interface transformed to tetragonal phase. It was further proved that interface modification with $\mathrm{Li}_{3} \mathrm{BO}_{3}$ can reduce chemical cross-contamination and improve physical bonding.

Introducing interfacial buffer layer, such as $\mathrm{Nb}, \mathrm{LiNbO}_{2}$, $\mathrm{BaTiO}_{3}$ was found to be an effective way to mitigate interface interdiffusion (Kato et al., 2014). By radio frequency (RF) magnetron sputtering, Kato et al. (2014) introduced a thin $\mathrm{Nb}$ layer $(10 \mathrm{~nm})$ on LLZO and then $\mathrm{LiCoO}_{2}$ was deposited on the $\mathrm{Nb}$-modified LLZO by PLD at $600^{\circ} \mathrm{C}$. In-situ synthesis of $\mathrm{LiCoO}_{2}$ by PLD guaranteed an intimate contact between cathode and solid electrolyte, while introducing $\mathrm{Nb}$ layer improved the interface performance by forming $\mathrm{Li}-\mathrm{Nb}-\mathrm{O}$ amorphous region. The $\mathrm{Li}^{+}$- conductivity of the amorphous $\mathrm{Li}-\mathrm{Nb}-\mathrm{O}$ region is high as $1 \times 10^{-6} \mathrm{~S} \mathrm{~cm}^{-1}$, which will facilitate $\mathrm{Li}^{+}$transport at interface (Glass et al., 1978). As a result, the mutual diffusion thickness is $40 \mathrm{~nm}$, which is much smaller than the $100 \mathrm{~nm} \mathrm{Li}^{+}$insulating $\mathrm{La}_{2} \mathrm{CoO}_{4}$ region without $\mathrm{Nb}$ modification (Kim et al., 2011). Kazyak et al. presented a thermal ALD (atomic layer deposition) process which can significantly lower the formation temperature of the cubic phase to $555^{\circ} \mathrm{C}$. The schematic processes and SEM images of LLZO products are illustrated in Figure 4B. Low melting compounds were also employed for good interfacial 

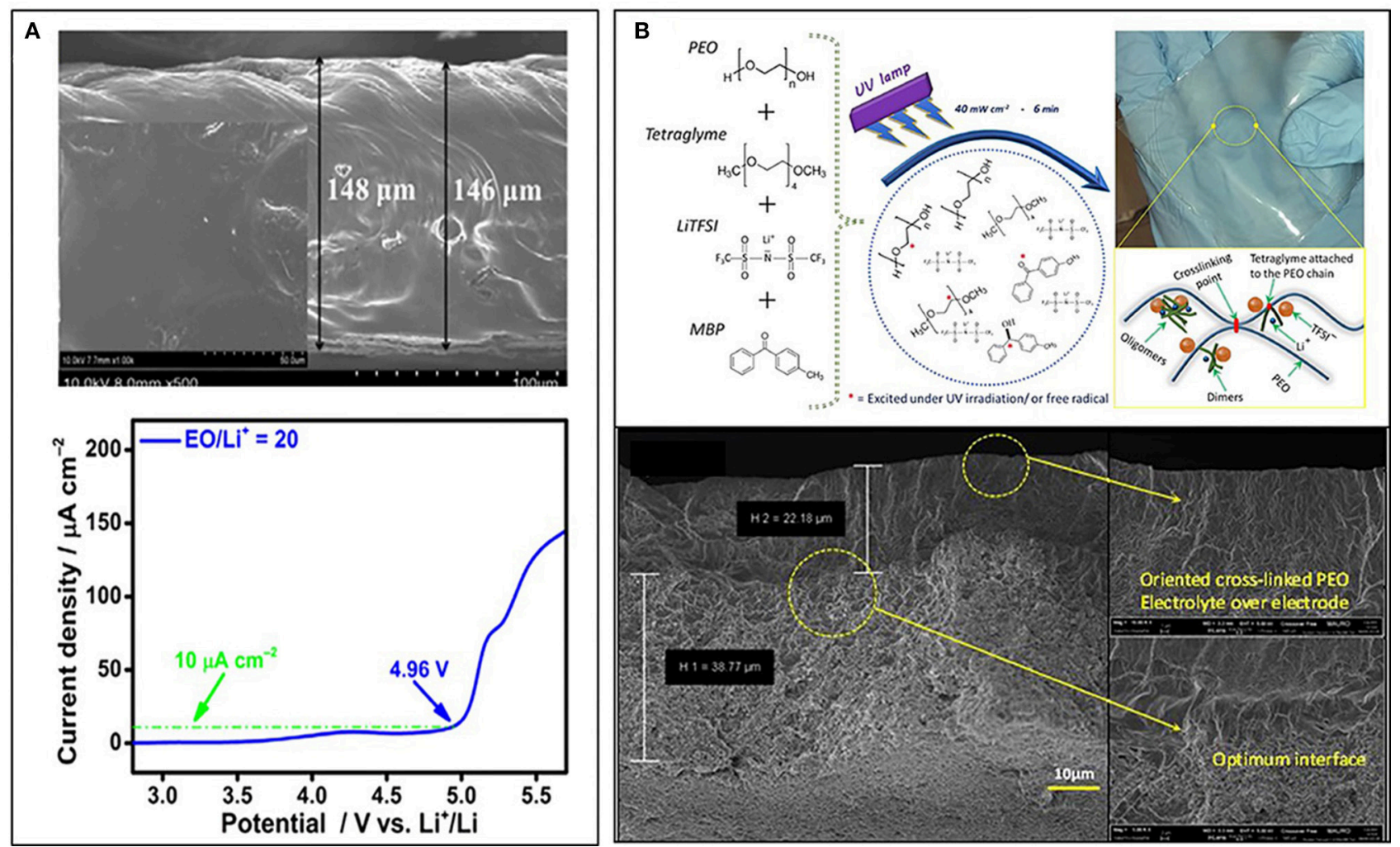

FIGURE 3 | (A) SEM image (up) and linear sweep voltammogram (down) for the membrane of the LiTNFSI/PEO (EO/Li $\left.{ }^{+}=20\right)$ blended polymer electrolyte. [Reprinted with permission from (Ma et al., 2016b). Copyright (2016) American Chemical Society]. (B) Interconnected PEO chains with hypothesized branched clusters of tetraglyme oligomers (top left) and the real aspect of a freshly prepared ISPE (top right); Cross-sectional FESEM images showing the optimum interface achieved after UV curing (down). [Reprinted with permission from (Porcarelli et al., 2016). Copyright (2016) Nature].

contact in solid state lithium batteries. Liu et al. used $\mathrm{Li}_{3} \mathrm{BO}_{3}$ (melting point $\mathrm{ca} .700^{\circ} \mathrm{C}, \mathrm{Li}^{+}$conductivity ca. $2 \times 10^{-6} \mathrm{~S}$ $\mathrm{cm}^{-1}$ ) as bonding aid for LCO/LLZO interface. Corresponding interface resistance reduced dramatically and electrochemical performance improved significantly. Yoshima et al. (2016) designed a gel to soften interface and achieved intimate contact at interface. LLZO coated with polyacrylonitrile (PAN)-based gel was prepared as electrolyte sheet, which reduced internal resistance of the whole battery. The assembled solid state lithium batteries exhibited good rate capability and cycling stability between -40 and $80^{\circ} \mathrm{C}$. Very recently, Han et al. reported an all-ceramic cathode-electrolyte by thermally soldering $\mathrm{LiCoO}_{2}$ and LLZO together with $\mathrm{Li}_{2.3-\mathrm{x}} \mathrm{C}_{0.7+\mathrm{x}} \mathrm{B}_{0.3-\mathrm{x}} \mathrm{O}_{3}$ solid electrolyte interphase can be spontaneously coated on both LLZO and $\mathrm{LiCoO}_{2}$ (Han et al., 2018). The simultaneous improvements in interfacial contact, (electro) chemical stability, ionic conductivity, and mechanical property of the all-ceramic cathode-electrolyte enabled an all solid state $\mathrm{Li} / \mathrm{LLZO} / \mathrm{LiCoO}_{2}$ battery with extremely high electrochemical performance.

Owing to the rigid ceramic nature, most solid oxide electrolytes face similar interfacial challenges when paired with solid cathode. The aforementioned interface modifying strategies can also be applied to diverse solid oxides electrolytes, except that typical solid oxide electrolyte are further hindered by other factors. Perovskite-type LLTO was firstly prepared by Inaguma et al. (1993) which exhibits low ionic conductivity across grain boundary (around $10^{-5} \mathrm{~S} \mathrm{~cm}^{-1}$ ) and poor stability with anode (instable below $1.8 \mathrm{~V}$ vs. $\mathrm{Li}^{+} / \mathrm{Li}$ ). As a result, most works on LLTO focus on improving ionic conductivity and chemical stability vs. Li anode (Chen and Amine, 2001; Kotobuki et al., 2011; Huang et al., 2016). $\mathrm{Li}_{1+\mathrm{x}} \mathrm{Al}_{\mathrm{x}} \mathrm{Ge}_{2-\mathrm{x}}\left(\mathrm{PO}_{4}\right)_{3}$ (LAGP) and $\mathrm{Li}_{1+\mathrm{x}} \mathrm{Al}_{\mathrm{x}} \mathrm{Ti}_{2-\mathrm{x}}\left(\mathrm{PO}_{4}\right)_{3}$ (LATP) are two common NASICON-type solid oxide electrolyte. Because of $\mathrm{Ti}^{4+}$ reduction, LATP suffers redox reaction at $2.5 \mathrm{~V}$ vs. $\mathrm{Li}^{+} / \mathrm{Li}$. Although LATP shows high ionic conductivity (Delmas et al., 1988), its incompatible with low potential anodes, especially $\mathrm{Li}$, confines its application in solid state lithium batteries. In these solid oxide electrolytes related research, interface softening, and in-situ synthesizing have also been carried out (Kim et al., 2017; Zhang et al., 2017b) and corresponding investigations are still in progress.

\section{Interface Between Cathode and Solid Sulfide Electrolyte}

Solid sulfide electrolytes are the derivatives of solid oxide electrolytes by substituting oxygen with sulfur. Since the electronegativity of $\mathrm{S}$ is less than $\mathrm{O}, \mathrm{Li}^{+}$binding energy is smaller and $\mathrm{Li}^{+}$can move more freely. Among all solid electrolytes, solid sulfide electrolyte exhibits the highest $\mathrm{Li}^{+}$conductivity. 


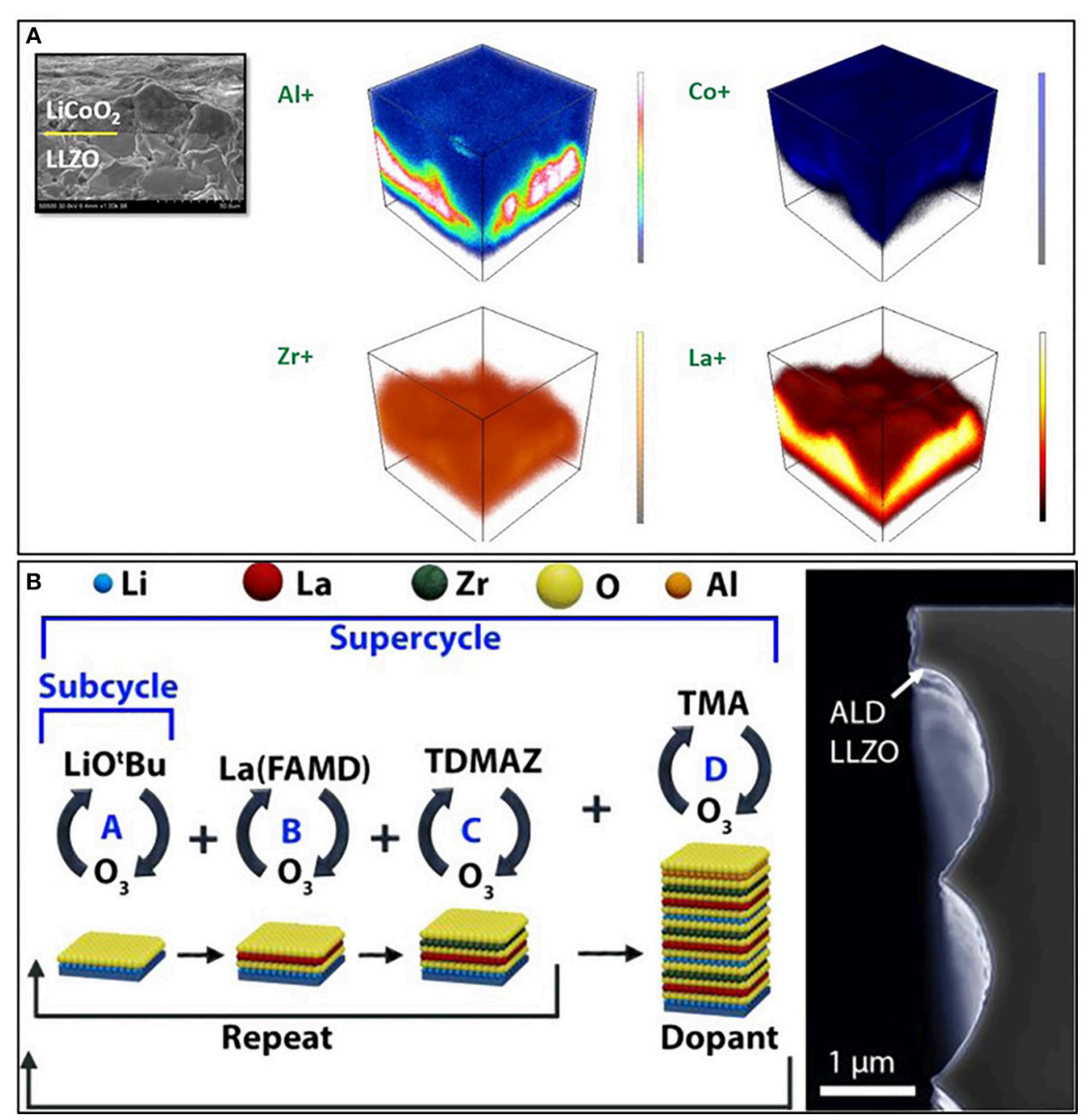

FIGURE 4 | (A) TOF-SIMS-enabled three-dimensional elemental maps of the $\mathrm{LiCoO}_{2} / \mathrm{Li}_{7} \mathrm{La}_{3} \mathrm{Zr}_{2} \mathrm{O}_{12}$ (LLZO) interface that is displayed in the inset SEM image. [Reprinted with permission from (Park et al., 2016). Copyright (2016) American Chemical Society]. (B) Schematic representation of an ALD super cycle composed of constituent binary ALD processes (left) and SEM image showing as-deposited ALD LLZO film (right). [Reprinted with permission from (Kazyak et al., 2017). Copyright (2017) American Chemical Society].

Another attractive feature of solid sulfide electrolyte is their mechanical property. These materials exhibit plastic deformation under mechanical pressure, and this softness makes it possible to prepare densely packed interface (Koerver et al., 2017). In recent years, the research focus of solid sulfide electrolyte is $\mathrm{Li}_{2} \mathrm{~S}$ $\mathrm{P}_{2} \mathrm{~S}_{5}$ based systems, which exhibit superior $\mathrm{Li}^{+}$conductivity, electrochemical stability, and mechanical properties. According to the composition difference, $\mathrm{Li}_{2} \mathrm{~S}-\mathrm{P}_{2} \mathrm{~S}_{5}$ system can be divided into binary solid sulfide electrolyte (composed of $\mathrm{Li}_{2} \mathrm{~S}$ and $\mathrm{P}_{2} \mathrm{~S}_{5}$, such as $\mathrm{Li}_{3} \mathrm{PS}_{4}, \mathrm{Li}_{7} \mathrm{P}_{3} \mathrm{~S}_{11}$ ) and ternary solid sulfide electrolyte (composed of $\mathrm{Li}_{2} \mathrm{~S}, \mathrm{P}_{2} \mathrm{~S}_{5}, \mathrm{MS}_{2}, \mathrm{M}=\mathrm{Si}, \mathrm{Ge}, \mathrm{Sn}$, such as $\mathrm{Li}_{10} \mathrm{GeP}_{2} \mathrm{~S}_{12}$ ). According crystallinity difference, the two kinds of SSEs can be further divided into glass, glass ceramic, and ceramic form solid electrolyte, which exhibit different performance in terms of ionic conductivity, chemical stability, and contact with solid electrode.
Owing to $\mathrm{Li}^{+}$chemical potential difference between oxide cathode and solid sulfide electrolyte, $\mathrm{Li}^{+}$may migrate from electrolyte to cathode, resulting in SCL at both sides. Due to the mixed ionic and electronic conductivity in oxide cathode, $\mathrm{Li}^{+}$gradient concentration can be compensated at cathode side. However, SCL will remain at electrolyte side due to the single ionic conductivity of electrolyte. The resulting SCL can wellimpede $\mathrm{Li}^{+}$transport and induce high polarization. SCL was firstly proposed by Wagner (1972) and extensively investigated on conduction type and conductivity change of composite materials, polycrystalline and heterojunctions (Liang, 1973; Maier, 1995; Bhattacharyya and Maier, 2004). With theoretically calculation, Haruyama et al. (2014) elucidated the characteristics of SCL between $\mathrm{LiCoO}_{2}$ and $\beta-\mathrm{Li}_{3} \mathrm{PS}_{4}$, the effect of $\mathrm{LiNbO}_{3}$ buffer layer interposition was also clarified. DFT calculation further revealed $\mathrm{Li}^{+}$-preferred adsorption at oxygen bridge sites, e.g., 
$\mathrm{CoO}_{6}$, and on Li layer, which may be the origin of deformed interface and SCL. Li chemical potentials based on vacancy formation energy indicate that the subsurface $\mathrm{Li}$ in sulfide electrolyte may transfer under electric field, suggesting that SCL grows immediately at the beginning of charging (Figure 5). Since the attractive sites on $\mathrm{LiCoO}_{2}$ surface disappear with insulating $\mathrm{LiNbO}_{3}$ layers attachment, the SCL at this interface is significantly suppressed. This result consistently explained SCL at atomic-scale and clearly indicated the effect of buffer layers. To eliminate SCL at the oxide cathode/SSE interface, oxide layer with high $\mathrm{Li}^{+}$conductivity, and chemical stability (mostly $\mathrm{LiNbO}_{3}$ ) is always introduced at interface and combined with other modification techniques (Kitaura et al., 2011; Haruyama et al., 2017; Koerver et al., 2017).

Even though solid sulfide electrolyte has moderate physical deformability, electrochemically driven mechanical failure also contributes to interfacial resistance increase and capacity fading. Koerver et al. evaluated the interfacial behavior for solid state lithium battery using nickel-rich NCM-811 cathode and $\beta$ $\mathrm{Li}_{3} \mathrm{PS}_{4}$ solid electrolyte (Koerver et al., 2017). Results suggest that the majority of passivating layer is developed during the first charge and present slow growth upon further cycling. It was further found that electrode-electrolyte contact lose occurs in first charging due to electrochemical contraction. The mechanical failure even deteriorates in the following cycles (Figure 6A) and lead to high polarization and capacity decay. In order to achieve and sustain intimate interface contact, different methods, and strategies have been developed and investigated. Sticking the supercooled liquid state of electrolyte on active material particles combined with a hot press was used to achieve an intimate electrode-electrolyte interface (Kitaura et al., 2011). In contrast to the interface formed by RT pressing, hot pressing at around $\mathrm{T}_{\mathrm{g}}$ may well-obtain intimate contact along with an interfacial layer between $\mathrm{LiCoO}_{2}$ and $80 \mathrm{Li}_{2} \mathrm{~S} \cdot 20 \mathrm{P}_{2} \mathrm{~S}_{5}$ glass electrolyte. $\mathrm{LiNbO}_{3}$ coating layer can be further introduced to suppress the reaction of $\mathrm{LiCoO}_{2}$ with the $80 \mathrm{Li}_{2} \mathrm{~S} \cdot 20 \mathrm{P}_{2} \mathrm{~S}_{5}$ glass solid electrolyte. Yao et al. (2016) reported a general interfacial architecture, i.e., $\mathrm{Li}_{7} \mathrm{P}_{3} \mathrm{~S}_{11}$ electrolyte particles (around $10 \mathrm{~nm}$ ) anchored on cobalt sulfide nanosheets, by insitu liquid-phase approach. The STEM-EDS elemental mapping of an individual nanocomposite in Figure 6B confirms that the cobalt sulfide- $\mathrm{Li}_{7} \mathrm{P}_{3} \mathrm{~S}_{11}$ nanocomposites are homogeneously distributed throughout the nanosheets and have an intimate contact. The obtained intimate contact contributed to an excellent rate capability and cycling stability. Similar intimate contacts could be achieved by sulfide electrolyte coating onto active materials to form a favorable interface (Ito et al., 2017). By Mixing $\mathrm{LiCoO}_{2}$ particles with different grain sizes during the electrolyte coating process, higher packing density pellets with less voids were obtained both before and after cycling which ensured fine networks of ionically conductive pathways. Moreover, Oh et al. (2016) discovered continuous LGPS decomposition at LGPS/acetylene black (AB) interface above $4.5 \mathrm{~V}$. The decomposition layer could also isolate the delithiated $\mathrm{LixNi}_{0.5} \mathrm{Mn}_{1.5} \mathrm{O}_{4}(\mathrm{x} \sim 0)$ from $\mathrm{Li}^{+}$and/or electron conduction channels in cathode composite, resulting in contact loss, and severe capacity fading upon cycling. The research demonstrates that suitable conductive additive and sulfide solid electrolyte are crucial to overcome the poor cycle performance of high-voltage solid state lithium batteries. Yoon et al. (2018) also investigated the interface between $\mathrm{Li}_{10} \mathrm{GeP}_{2} \mathrm{~S}_{12}$ and diverse carbon conductive agents in solid state lithium batteries and confirmed the solid electrolyte decomposition and surface degradation during cycle.

\section{Solid Composite Electrolyte Design and Interface Optimization}

Solid composite electrolyte is a subset of polymer electrolytes by dispersing electrochemically inert fillers, such as $\mathrm{Al}_{2} \mathrm{O}_{3}$ and $\mathrm{TiO}_{2}$ nanoparticles or inorganic solid electrolyte into polymer electrolyte. (Weston and Steele, 1982; Croce et al., 1998, 1999) These composite electrolytes have excellent mechanical stability (due to the ceramic fillers into polymer network) and high ionic conductivity (promoted by the high surface area of the dispersed fillers). Due to the absence of liquid components and interfacial stabilizing action from dispersed fillers, composite electrolyte offers wide electrochemical stability window (Croce et al., 1999). With advantages such as high ionic conductivity, wide electrochemical stability window, favorable interface mechanical properties, composite electrolytes have attracted extensive attention.

The inorganic fillers in solid composite electrolytes could be oxides without $\mathrm{Li}^{+}$conducting ability, such as $\mathrm{Al}_{2} \mathrm{O}_{3}$, $\mathrm{TiO}_{2}, \mathrm{SiO}_{2}$, etc. (Lin et al., 2016; Pal and Ghosh, 2018) and other solid electrolytes, such as LLZO, LAGP, LGPS, etc. (Zhao et al., 2016; Chen et al., 2017, 2018a,b; Zhai et al., 2017). In 2016, Lin et al. introduced a novel in-situ synthesis of a $\mathrm{SiO}_{2}$ filler inside $\mathrm{PEO}$ polymer. Much stronger chemical/mechanical interactions between $\mathrm{SiO}_{2}$ nanospheres and PEO chains can be obtained, which significantly suppresses PEO crystallization and facilitates polymer segmental motion for $\mathrm{Li}^{+}$conducting ( $\mathrm{Lin}$ et al., 2016). Two possible interaction mechanisms are shown in Figure 7A, including chemical bonding between PEO chains and hydroxyl groups on $\mathrm{MUSiO}_{2}$ and mechanical wrapping of $\mathrm{PEO}$ chains during $\mathrm{MUSiO}_{2}$ spheres growth. At the same time, electrochemical stability window can be largely extended up to $5.5 \mathrm{~V}$, much wider than ex-situ CPE and ceramic-free CPE (Figure 7A). The improvement of electrochemical stability indicates that the adsorption effect on anion is much stronger in in-situ CPE, which suppresses anodic decomposition at high potential (Park et al., 2003).

Garnet type LLZO electrolyte, has been widely studied as kind of filler in PEO matrix (Zheng et al., 2016; Chen et al., 2017, 2018a; Zhang et al., 2017a). Recently, Chen et al. (2018a) proposed a synergistic-composite approach to fabricate flexible solid state lithium batteries using $\mathrm{PEO}$-based composite cathode layers (filled with $\mathrm{LiFePO}_{4}$ particles) and composite electrolyte layers (filled with Al-LLZTO particles) which exhibits a wide electrochemical stability window $\sim 6 \mathrm{~V}$, much wider than pure PEO. The all-composite approach is favorable for improving both mesoscopic and microscopic interfaces (Figure 7B) inside solid state lithium batteries and may provide a new toolbox for solid state lithium batteries design and fabrication. The interface 


\section{Equilibrium (Atomic structure)}
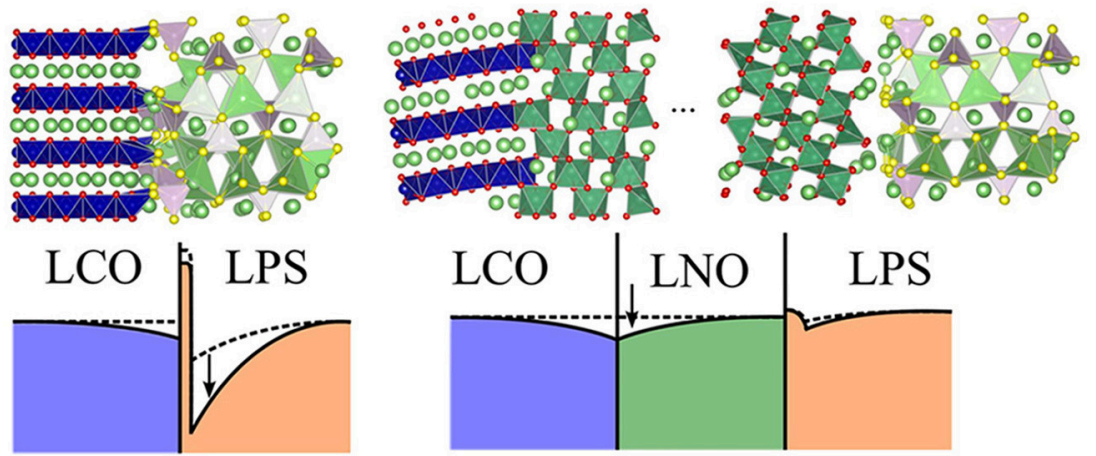

\section{Initial Stage of Charging (Li ion concentration)}

FIGURE 5 | Comparison of atomic structures (at equilibrium state) and Li-ion concentration (at initial stage of charging) at the LiCoO $2 / \mathrm{Li}_{3} \mathrm{PS}_{4}$ interfaces without (left) and with (right) $\mathrm{LNbO}_{3}$ buffer layer. [Reprinted with permission from (Haruyama et al., 2014). Copyright (2014) American Chemical Society].
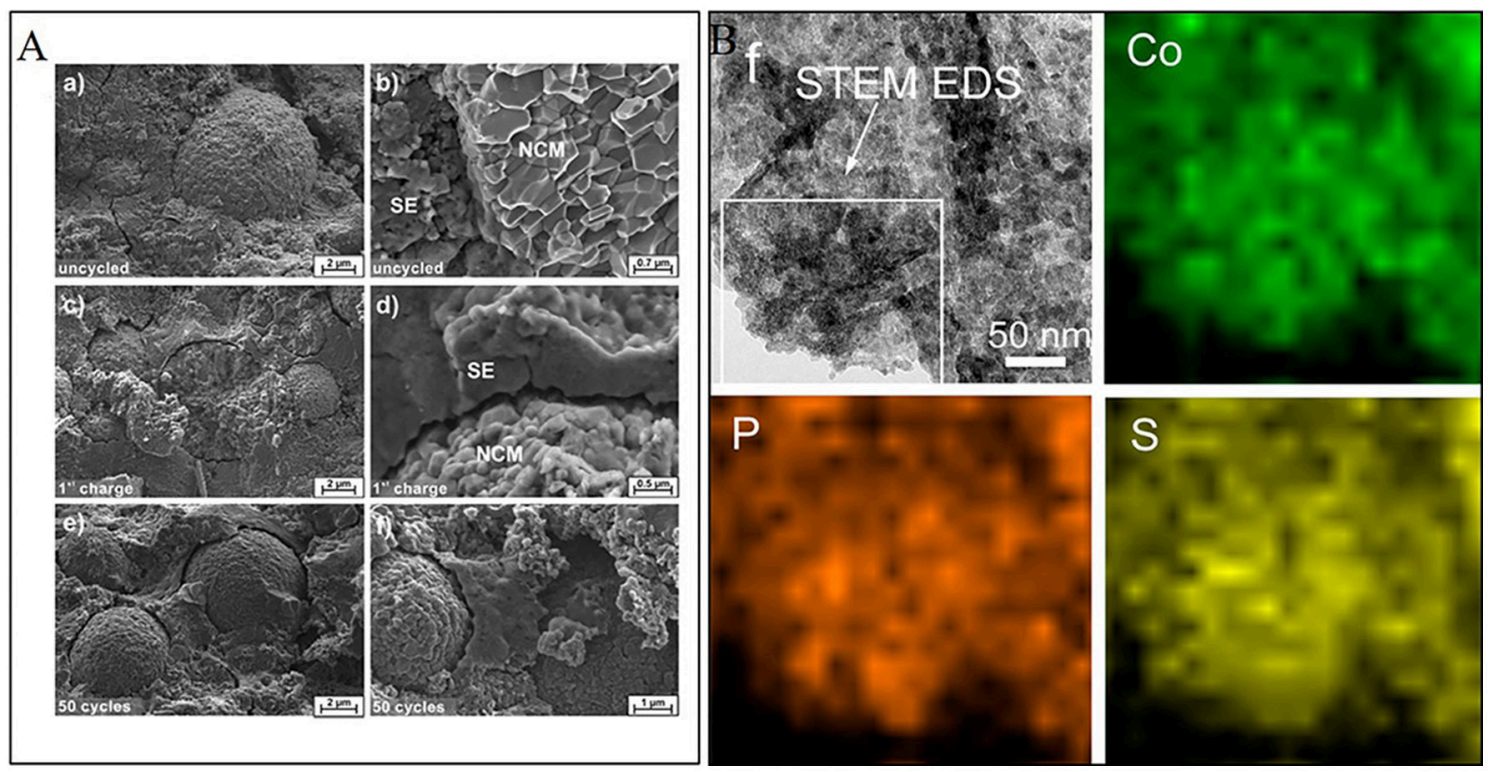

FIGURE 6 | (A) Scanning electron micrographs of the cathode composite of NCM811 and $\beta$ - Li $i_{3} P S_{4}$ : (a,b) before cycled; (c,d) after single charging to $4.3 \mathrm{~V}$ vs. Li/Li+ at $0.1 \mathrm{C}$; (e,f) after 50 full battery cycles in the discharged state. [Reprinted with permission from (Koerver et al., 2017). Copyright (2017) American Chemical Society]. (B) STEM EDS elemental mapping images of cobalt sulfide-Li7 $\mathrm{P}_{3} \mathrm{~S}_{11}$ nanocomposites. [Reprinted with permission from (Yao et al., 2016). Copyright (2016) American Chemical Society].

between composite cathode and composite electrolyte layers may keep its structural integrity albeit the large volumetric change during cycling (Chen et al., 2017). Since the synergeticcomposite electrolyte combines the virtues of two components, compositing stands a chance in building favorable interfaces, and further realizing high energy density solid state lithium batteries. Chen et al. (2018a) prepared composite ceramic/polymer solid electrolyte containing garnet $\mathrm{Li}_{6.4} \mathrm{La}_{3} \mathrm{Zr}_{1.4} \mathrm{Ta}_{0.6} \mathrm{O}_{12}$ (LLZTO), $\mathrm{PEO}$, and Lithium bis(trifluoromethanesulfonyl)imide (LiTFSI).
The composite solid electrolyte possesses high self-standing and flexibility, which exhibits electrochemical stability window up to $5.0 \mathrm{~V}$ vs. $\mathrm{Li} / \mathrm{Li}^{+}$. The assembled solid-state $\mathrm{LiFePO}_{4} \mid \mathrm{Li}$ batteries with electrolytes from "ceramic-in-polymer" to "polymer-in-ceramic" exhibit excellent cycling stability and wide electrochemical stability window (more than $5.0 \mathrm{~V}$ vs. $\left.\mathrm{Li}^{+} / \mathrm{Li}\right)$. The "ceramic-in-polymer" electrolyte exhibits a greater flexibility (Figure 7C) and lower cost, while "polymerin-ceramic" electrolyte shows higher mechanical strength 


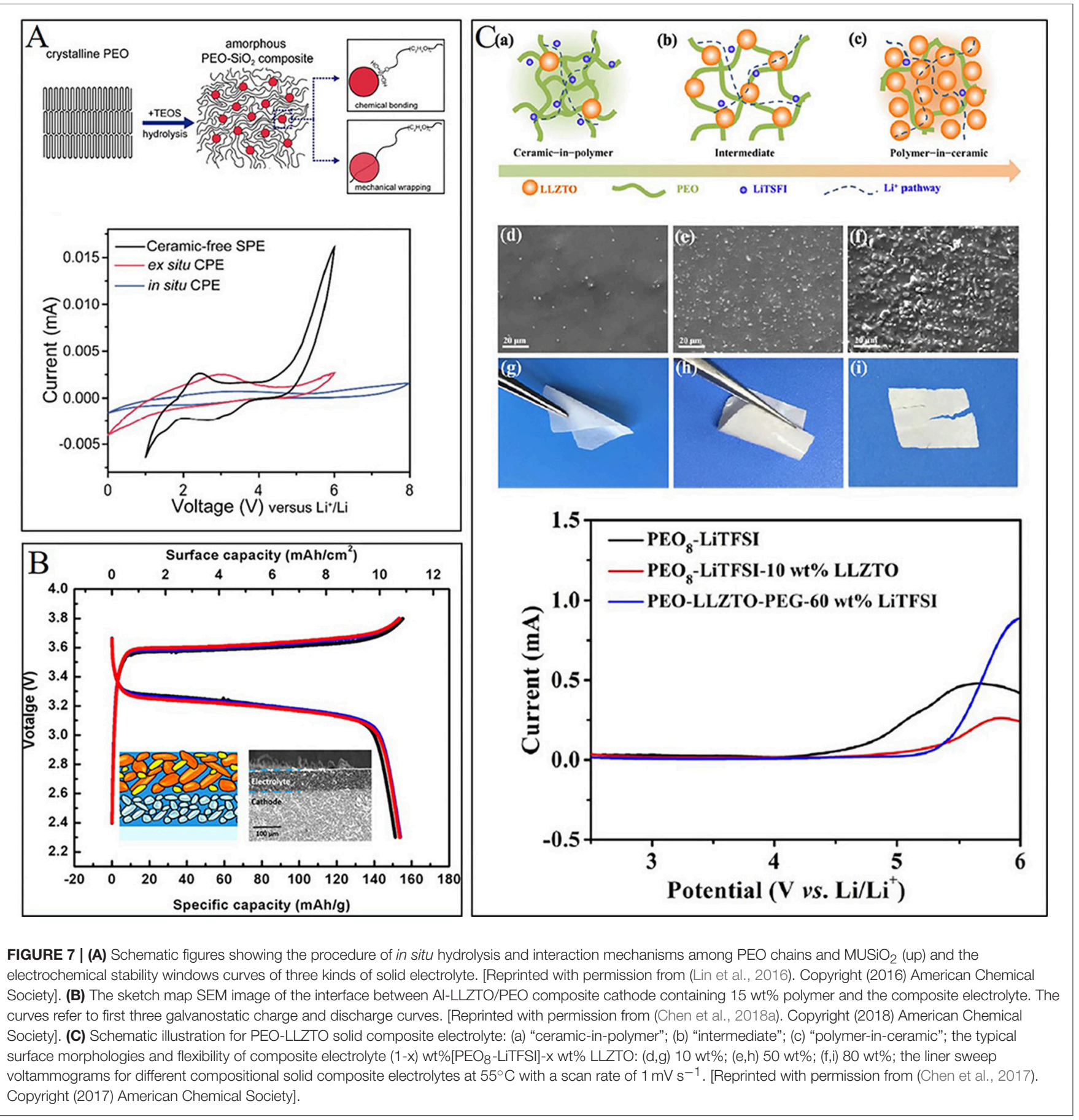

and safety but brittler for bend cracks formation. Hence, by varying the composition of composite electrolyte, different properties will be obtained for special applications. Apart from LLZO, other oxide solid electrolyte, such as LATP and LAGP are also widely combined with PEO in order for better composite solid electrolyte (Wang et al., 2017b; Zhai et al., 2017).

Compared with TM oxide particles, solid sulfide electrolyte, such as LGPS and $\mathrm{Li}_{3} \mathrm{PS}_{4}$, exhibits fast ionic conductivity (Han et al., 2011; Haruyama et al., 2014; Kato et al., 2016). As a result, solid sulfide electrolyte incorporate into PEO matrix can provide excellent $\mathrm{Li}^{+}$conducting channel. Zhao et al. fabricated SPE membranes comprised of LGPS and PEO matrix. The optimal composite membrane exhibits high ionic conductivity $\sim 1.21$ $\times 10^{-3} \mathrm{~S} \mathrm{~cm}^{-1}$ at $80^{\circ} \mathrm{C}$ and wide electrochemical window of 0-5.7 V (Zhao et al., 2016). Instead of simply mixing ceramic particles with polymers, the same group prepared $\mathrm{PEO} / \mathrm{Li}_{3} \mathrm{PS}_{4}$ hybrid polymer electrolyte via new in-situ approach (Chen et al., 
2018b). The optimal electrolyte of PEO-2\% vol $\mathrm{Li}_{3} \mathrm{PS}_{4}$ presents the highest $\mathrm{Li}^{+}$conductivity and widest electrochemical window. In anodic process, in-situ prepared electrolyte shows no anodic current until $5.1 \mathrm{~V}$, while corresponding voltage for mechanicalmixed electrolyte and PEO are $4.9 \mathrm{~V}$ and $4.6 \mathrm{~V}$, respectively, as reported in their article. The differences in ionic conductivity and stability may originate from more homogeneous dispersion of fillers in PEO by in-situ preparation than by mechanical-mixing.

According to the mechanical properties including flexibility, deformability, and strength of the aforementioned four kinds of electrolytes, the different interface performance and modifications at cathode side are summarized in Table $\mathbf{1}$.

\section{ADVANCED SOLID-SOLID INTERFACE CHARACTERIZATION TECHNIQUES}

As discussed above, the interface behaviors play an important part in determining the final solid state lithium battery parameters and performances, including internal resistance, kinetic response, and cycle stability etc. However, the buried solid-solid interfaces in solid state lithium batteries are extremely difficult to investigate directly, and present knowledge on interfacial reactions and interfacial kinetics is still deficient. As a result, it is increasingly important and urgent to develop novel characterization techniques for more detailed understanding into the interface behavior ( $\mathrm{Hu}$ et al., 2006). Note that the solid electrolyte-cathode interface involves several aspects correlated with each other, including lattice structure, electronic band structure, and chemical/electrochemical stability. The dynamic $\mathrm{Li}$ shuttle back and force across the interface further makes the interface behavior more complicated within time and voltage domain. Hence, advanced characterization techniques with in-situ and atomic-scale resolution are strongly necessary to gain more insights into the complex interface processes (Zheng et al., 2014; Lin et al., 2017). So far, diverse advanced characterizations have been utilized by different research groups worldwide and significant information have been obtained. These research works provide valuable perspective of interface performance and have profound guiding significance for designing more favorable interfaces in superior solid state lithium batteries.

Since a significant reason for interfacial instability is the abrupt change of electric potential across the cathode-electrolyte interface, dynamic observation of the potential profiles would help identify sources of typically large interfacial resistance. Ogumi' group contributed a lot in studying the potential distribution and interface stability mechanisms in solid state lithium batteries (Yamamoto et al., 2010, 2012; Okumura et al., 2011). With this objective, $\mathrm{EH}$ (quantitative electron holography) combined with EELS (electron energy loss spectroscopy) was used to directly observe the potential distribution at the $\mathrm{LiCoO}_{2} / \mathrm{Li}_{1+\mathrm{x}+\mathrm{y}} \mathrm{A}_{\mathrm{y}} \mathrm{Ti}_{2-\mathrm{y}} \mathrm{Si}_{\mathrm{x}} \mathrm{P}_{3-\mathrm{x}} \mathrm{O}_{12}$ interface (Yamamoto et al., 2010). Results showed the $\mathrm{Li}^{+}$and electron typical distribution of the measured potential near the cathode-electrolyte interface during charging and the origin of the shift of the electronic band structures. This research identified the sources of reaction resistance and kinetic factors in solid state lithium battery. $\mathrm{EH}$ also clearly show how the metallic lithium is formed inside the solid electrolyte during the initial charging process of the solid state lithium battery (Yamamoto et al., 2012). Results showed that the smooth potential distribution at the electrode/solid electrolyte interface leads to the low interfacial resistance.

With the unique sensitivity and operability in SCL detection, AFM was performed to better understand the potential distribution at the cross section of particles (Liang et al., 2018). By introducing LATP coating at cathode surface, the $\mathrm{LiNi}_{0.6} \mathrm{Co}_{0.2} \mathrm{Mn}_{0.2} \mathrm{O}_{2} /$ poly(ether-acrylate) (ipn-PEA) interface realized a mitigated polarization and excellent kinetic performance. The significantly improved interface dynamics is attributed to a weakened SCL or a gradual slope of potential formed at the interface, verified by AFM interfacial potential measurements, which relieves polarization, alleviates side reactions, and enhances cycling stability and dynamic properties (Figure 8).

Apart from interfacial potential research techniques, advanced characterizations adopted to investigate structural and chemical stability of the interface also promote the mechanism understanding of the interface behavior. In/ex situ characterization techniques including spectroscopy, microscopy, and diffractometry are widely used to monitor the structural evolution and chemical reactions at the interface.

Spectroscopy including XAS (X-ray absorption spectroscopy), XPS (X-ray photoelectron spectroscopy), NMR (Nuclear Magnetic Resonance Imaging), etc. in an in-situ mode with high spatial resolution play important role in the solid-solid interface understanding. Okumura et al. (2011) developed a depth-resolved XAS to directly observe the chemical state and local structure at the $\mathrm{LiCoO}_{2}$ /LATP interface with/without $\mathrm{NbO}_{2}$ modification layer. XAS results revealed that the introduction of $\mathrm{NbO}_{2}$ layer is effective for restricting the large $\mathrm{Co}-\mathrm{O}$ bond change at the interface during delithiation. As a result, the charge transfer process is smoother owing to a relieved interface stress. Wenzel et al. developed in-situ XPS by using the internal argon ion gun of the instrument which was adopted to sputter a metallic target (Wenzel et al., 2015). The chemical stability of the LLTO/metallic lithium interface was investigated. The same in-situ XPS method was adopted by the same group to investigate the interface between lithium metal and $\mathrm{Li}_{10} \mathrm{GeP}_{2} \mathrm{~S}_{12}$. XPS recorded the decomposition products which revealed the formation of lithium sulfide, lithium phosphide, and germanium-lithium alloy/germanium metal (Figures 9A,B) (Wenzel et al., 2016). In situ NMR is also widely used to study the lithium distribution in solid lithium batteries (Bhattacharyya et al., 2010; Nakayama et al., 2010; Wang et al., 2014; Romanenko et al., 2016; Chien et al., 2018). Very recently, three-dimensional ${ }^{7} \mathrm{Li}$ magnetic resonance imaging (MRI) is employed to examine $\mathrm{Li}$ distribution homogeneity in solid electrolyte $\mathrm{Li}_{10} \mathrm{GeP}_{2} \mathrm{~S}_{12}$ within symmetric $\mathrm{Li} / \mathrm{Li}_{10} \mathrm{GeP}_{2} \mathrm{~S}_{12} / \mathrm{Li}$ batteries by Chien et al. (2018) (Figure 9C). The three-dimensional Li distribution revealed that the significant $\mathrm{Li}$ loss at interfaces is mitigated via PEO coating (Figure 9D) (Chien et al., 2018). This study demonstrates a powerful tool for non-invasively monitoring the 
TABLE 1 | Interfacial challenges exist in cathode-solid electrolyte systems according to the different characteristics of the four types of solid electrolytes and the corresponding solutions, recent advances and limitations still exist.

\begin{tabular}{lll}
\hline Interfaces & $\begin{array}{l}\text { Interfacial mechanical } \\
\text { performance }\end{array}$ & $\begin{array}{l}\text { Interfacial chemical } \\
\text { stability }\end{array}$ \\
\hline $\begin{array}{ll}\text { Cathode-Solid polymer } \\
\text { electrolyte interface }\end{array}$ & $\begin{array}{l}\text { Excellent elasticity and } \\
\text { deformability promote } \\
\text { favorable interface contact } \\
\text { Poor strength cannot block } \\
\text { Li dendrites }\end{array}$ & $\begin{array}{l}\text { PEO-based SPE is not } \\
\text { stable above 4.0 V (Croce } \\
\end{array}$ \\
& &
\end{tabular}

Cathode-solid oxide electrolyte interface

Cathode-solid sulfide electrolyte interface

Cathode /solid composite electrolyte interface
High strength properties can Stable up to 6V (Li et al., partially block dendrite 2015; Thangadurai et al., Poor flexibility lead to a poor 2015) solid-solid contact Dendrite can grow along grain boundaries
High-temperature handling $\left(>500^{\circ} \mathrm{C}\right.$ ) to may lead to elements interdiffusion and form transition layer
Reasonable strength and decent deformability Poor elasticity lead to contact loss upon periodic cathode expanding and shrinking

Combine the virtues of both polymer and ceramic with both reasonable strength and flexibility, promising to obtain favorable contact
High Li chemical potential leads to a space charge layer when matched with oxide cathodes Electrochemically unstable when contacted with high-voltage cathode

By adding inorganic fillers in PEO based solid electrolyte, the anti-oxidation property at high voltage is still under discussion even various studies reported high electrochemical window
Solutions and advances

(a) Optimization of Li salts (Zhang et al., 2011; Ma et al., 2016b)

(b) Polymer matrix modification such as copolymerization, branching and crosslinking (Tong et al., 2014; Porcarelli et al., 2016)

(c) Gel/ plasticized polymer electrolyte (Manuel Stephan, 2006; Zewde et al., 2018)

(d) Cathode coating (Ma et al., 2017; Yang et al., 2018)

(a) Surface coating (Han et al., 2017)

(b) Co-sintering (Wakayama et al., 2016)

(c) In-situ synthesized electrolyte layer (Yoshima et al., 2016; Kazyak et al., 2017)

(d) Interface softening (Seino et al., 2011; Sakuda et al., 2012; Liu et al., 2016)

(e) Interface buffer layers (Kato et al., 2014; Park et al., 2016)

(f) Amorphous cathode (Matsuyama et al., 2016; Nagao et al., 2017)

(a) Interface buffer layer to mitigate SCL (Haruyama et al., 2014; Koerver et al., 2017)

(b) Hot press, in-situ synthesis and sulfide electrolyte coating onto active materials to obtain intimate contact (Kitaura et al., 2011; Yao et al., 2016; Ito et al., 2017)

By regulating the composition of composite electrolyte, solid electrolytes with different performance will be obtained to adapt to different requirements

\section{Limitations still exist}

(a) Performance matched with highvoltage cathode such as $\mathrm{LiCoO}_{2}$ and $\mathrm{Li}_{2} \mathrm{MnO}_{4}$ is still poor

(b) The weaknesses of liquid electrolyte still exist in gel system, such as flammable property

(c) Short-circuit concerns

(a) Even intimate contact could be achieved at pristine state, contact loss will happen upon cycling during to the rigid ceramic nature

(b) High interface resistance prohibits thicker cathode layer and high capacity battery as a result

Contact loss upon cycling is still an unsolved problem which makes external pressure necessary

Drawbacks exist in single solid electrolyte system such as poor stability of SPE, SCL in solid sulfide electrolyte and poor

flexibility of solid oxide may still exist when these components contact cathode A novel solid electrolyte with high ionic conductivity, chemical stability Compatibility with cathode is still a long way to go
Li distribution at the interfaces and in the bulk of solid state lithium batteries as well as a convenient strategy for improving interfacial stability. As mentioned in chapter 3, TOF-SIMS can also be used to study the interface element distribution around the interface in solid state lithium batteries (Park et al., 2016).

Microscopy [e.g., TEM (transmission electron microscope), STEM (scanning transmission electron microscopy), etc.] techniques are powerful tools to investigate the structural and chemical stability of solid electrode/solid electrolyte interface. Wang et al. (2016b) used in situ STEM-EELS with high spatial resolution observed the interfacial phenomena of $\mathrm{LiCoO}_{2} / \mathrm{LiPON}$ with a nanoscale resolution (Figure 9E). An unexpected structurally disordered interfacial layer was discovered without cycling. The interfacial layer accumulates lithium and evolves to rock salt $\mathrm{CoO}$ after cycling along with
$\mathrm{Li}_{2} \mathrm{O}$ and $\mathrm{Li}_{2} \mathrm{O}_{2}$ formation (Figure 9F) (Wang et al., 2016b). Rapid capacity decay or even cathode inactivation will happen along with the thickening of this layer. In situ STEM was also introduced to study the interface stability of lithium metal/solid electrolyte (Ma et al., 2016a). Gong et al. (2017) designed an in situ atomic-scale TEM observation of electrochemical delithiation induced structure evolution of $\mathrm{LiCoO}_{2}$ cathode in solid state lithium batteries, which provides atomic-scale structure information for designing better solid state lithium batteries.

In situ diffractometry including XRD (X-ray diffraction) and ND (neutron diffraction) were also widely used to monitor the structural change upon cycling in solid state lithium batteries (Shin et al., 2014; Wang et al., 2017a; Hu et al., 2018). Wang et al. developed an in situ ND technique to monitor the Li distribution 

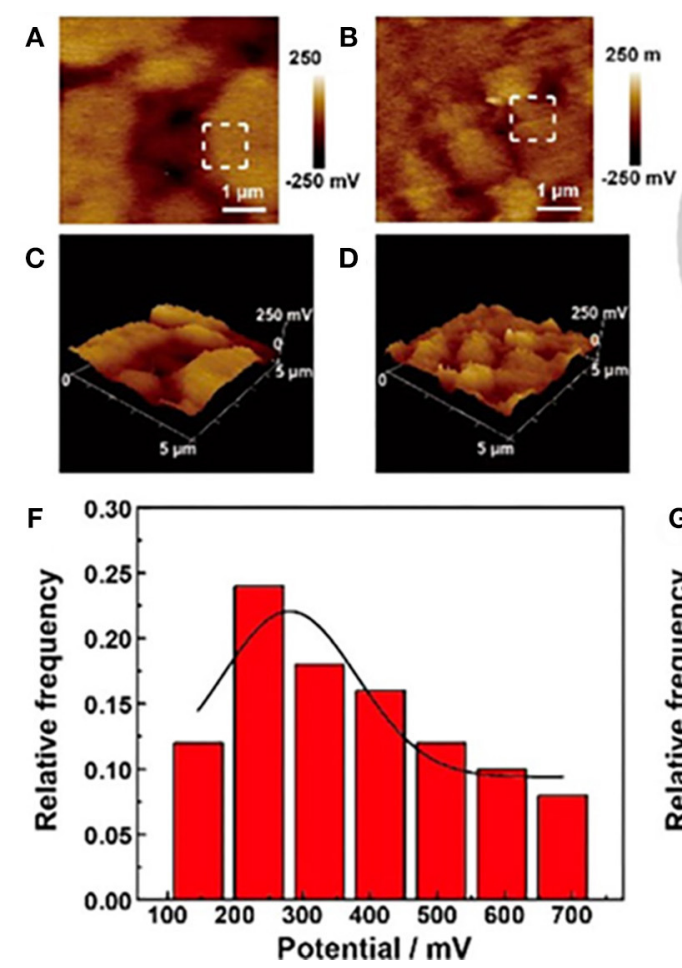
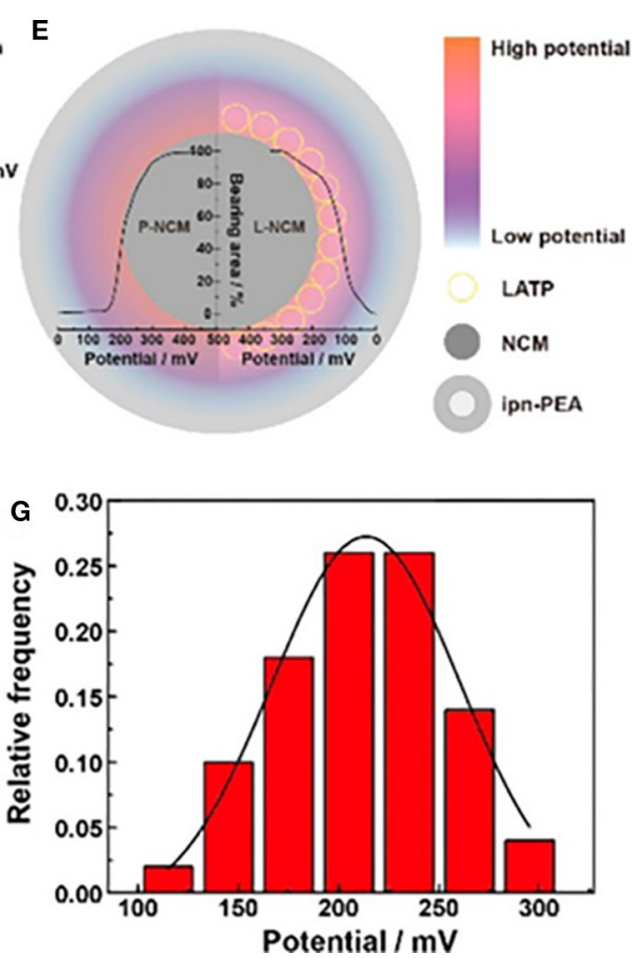

FIGURE 8 | AFM interfacial potential images and the corresponding 3D images of (A,C) pristine-NCM and (B,D) LATP coated-NCM. (E) Schematic diagram with bearing analysis and potential distribution of the two cathodes. The Gauss statistic distribution histograms of interfacial potential for (F) pristine-NCM and (G) LATP coated-NCM. [Reprinted with permission from (Liang et al., 2018). Copyright (2018) American Chemical Society].

and transport in garnet-based solid-state cells during cycling (Figures 9G,H) (Wang et al., 2017a). When Li is deposited outside the reversible layer, it becomes "dead lithium". A 3D mixed electron-ion conductive framework is preferred as a $\mathrm{Li}$ metal host to increase the contact area, shorten the Li diffusion distance, and overcome the anticipated volume change.

\section{CONCLUSION AND PERSPECTIVES}

This review provides a brief survey of recent research and development with respect to cathode-solid electrolyte interfaces in solid-state lithium batteries. We summarized the basic electrochemistry and principle at cathode-solid electrolyte interface, fundamental factors inducing interface challenges, and research progresses on building better interfaces. The interface issues in solid organic electrolytes, solid inorganic electrolytes, and solid composite electrolytes were reviewed and corresponding solutions are summarized on the basis of intrinsic characteristics of different solid electrolyte.

The interface degradation in solid state lithium battery may stem from the chemical/electrochemical stability and mechanical stability. Poor chemical/electrochemical stability between cathode and electrolyte may cause electrolytes decomposition or elements interdiffusion and transition region formation. The fundamental mechanism of interfacial chemical instability lies in the distribution of ionic electrochemical potential and inner electric potential gradient. Electronic and ionic conductivity of the transition region fundamentally determine whether a stable interface will form or not. An ionic conducting and electronic insulating transition layer will prevent further oxidation/reduction of electrolytes and SCL growth. While a mixed ionic and electronic conducting layer will result in continuous reactions and transition region growth.

To improve the chemical stability at cathode/SPE interface, different strategies can be adopted for different types of solid electrolytes. Modification of PEO matrix and lithium salts, adding proper plasticizer, cathode coating, and compositing with inorganic fillers are favorable for SPE and have made great progress. Solid oxide electrolytes are fairly stable with cathode compared with other solid electrolytes. While interdiffusion will take place along with high temperature dealing during in-situ synthesis, co-sintering, and deposition. Proper modification layer is needed to guarantee both chemical stability and intimate contact. Solid sulfide electrolytes, which suffer from SCL problem and decomposition reactions, should introduce other surface modifying method on the basis of introducing a proper buffer layer to eliminate SCL.

In addition to the chemical instability, it's difficult for inorganic solid electrolytes especially solid oxide electrolyte to maintain intimate contact with cathode due to the rigid ceramic nature. To achieve good mechanical contact, strategies such as surface coating, co-sintering, in-situ synthesis of electrolyte layer, interface softening, interface buffer layers were employed. By interface modification, the above issues are mitigated to a 

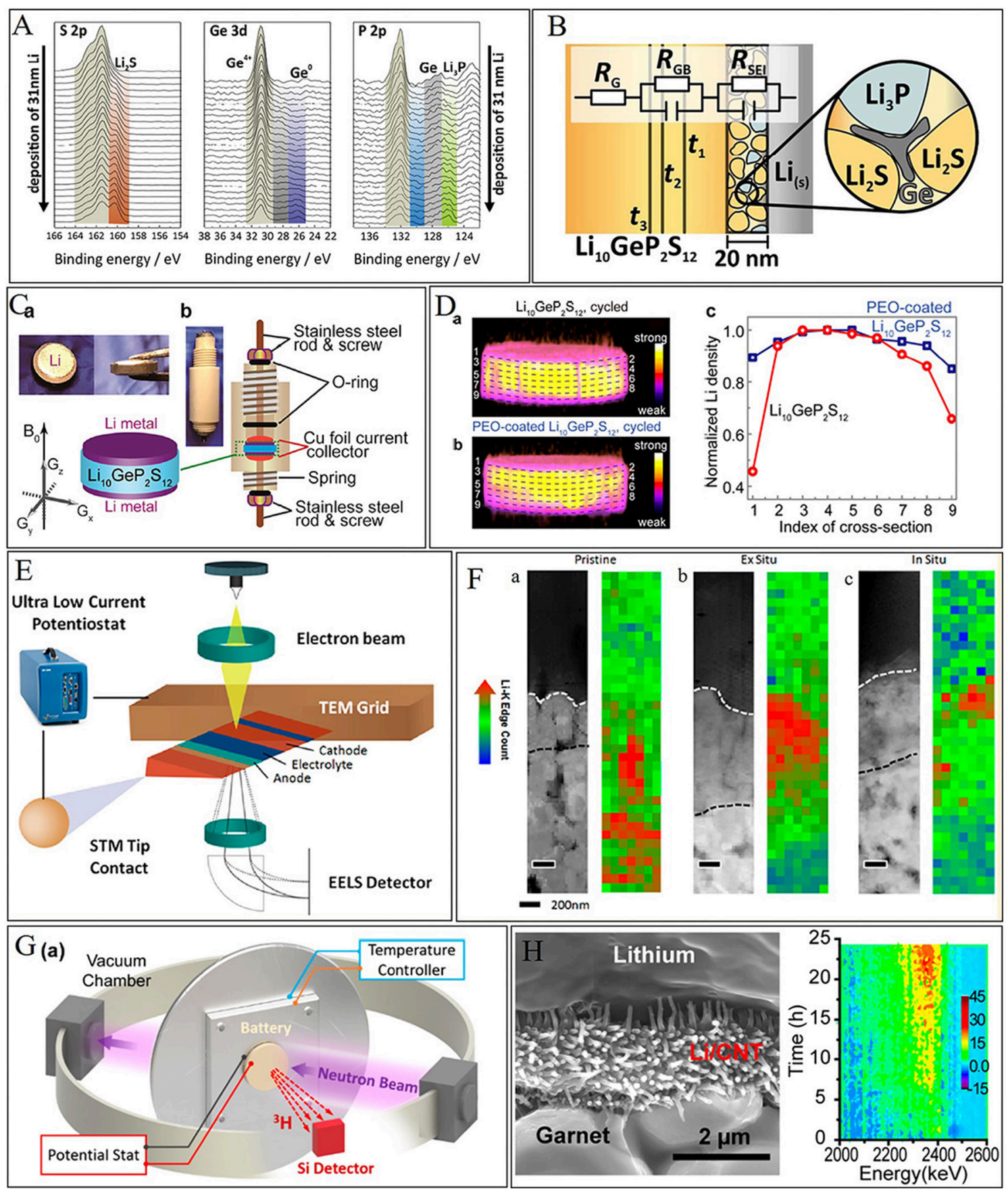

FIGURE 9 | In situ characterization techniques for solid-solid interface stability. (A) in situ XPS recorded during deposition of Li metal on LGPS. With increasing Li deposition time, LGPS decomposes. (B) Schematic of the interphase formation at the Li/LGPS interface according to the XPS result. [Reprinted with permission from (Wenzel et al., 2016). Copyright (2016) American Chemical Society]. (C) Pictures and schematic of a cylindrical cell for MRI. (D) Li density profiles at different depths of electrochemically cycled LGPS pellets. [Reprinted with permission from (Chien et al., 2018). Copyright (2018) American Chemical Society]. (E) Schematic of in situ TEM biasing of nanobattery. (F) STEM image and EELS characterization. (a-c) HAADF image of the nanobattery stack along with Li K-edge concentration mapping of (a) pristine, (b) ex situ, and (c) in situ samples with scale bar represents $200 \mathrm{~nm}$. [Reprinted with permission from Wang et al. (2016b). Copyright (2016) American Chemical Society]. (G) Schematic of the NDP system. (H) 2D projection of the NDP spectra collected at 5 min intervals during cycling. [Reprinted with permission from (Wang et al., 2017a). Copyright (2017) American Chemical Society]. 
large degree, while a novel solid electrolyte with high ionic conductivity, chemical stability, compatibility with both cathode, and anode is still a long way to go.

In order to gain more insights into interface behavior, advanced characterization techniques are necessary, particularly with time, and atomic-scale resolution. Recent investigations and progress obtained from diverse advanced characterization techniques are summarized here. Note that interface phenomena involve multiscale and multidimensional properties change, the combination of diverse technologies may help provide comprehensive understanding. Electron holography and internal potential from AFM help uncover the electric potential profiles across interface. Spectroscopy including XAS, XPS, and NMR directly probes the chemical state and local structure. Microscopy including TEM and STEM provide atomic arrangement at interface structure. While XRD and ND can well-monitor structure evolution upon battery cycle. MRI and TOF-SIMS may further build up 3-dimensional distribution of particular elements. Despite valuable information obtained from these tools, here we highlight that the wider cooperation between diverse techniques will provide stronger support to the final clarification of interface related phenomena.

According to detailed analysis of interface issues and solutions for different systems, we further conclude that to realize a favorable interface, a hybrid solution should be employed to achieve both mechanical and chemical/electrochemical stability. It's hard for one kind of solid electrolyte to combine both excellent elasticity to achieve intimate contact and favorable strength to resist lithium dendrite formation. On the one hand, an elastic and transformable electrolyte which could shrink and expand in pace with cathode is essential for solid state lithium batteries, such as SPE and special solid sulfide electrolyte. Note that even deformable sulfide electrolyte could not keep contact with electrode particles upon cycling, therefore other strategies such as in-situ synthesis and surface coating may serve as proper ways to modify solid sulfide electrolytes. In order to obtain pristine intimate contact before cycling, strategies such as flashburning, interface sintering, deposition method, and in situ polymerization are necessary. Meanwhile, to relieve the strain and stress resulted from the shrinkage and expansion of cathode material, nano-sized solid electrolyte and electrode may serve

\section{REFERENCES}

Aono, H., Sugimoto, E., Sadaoka, Y., Imanaka, N., and Adachi, G. (1990). Ionic conductivity of solid electrolytes based on lithium titanium phosphate. J. Electrochem. Soc. 137, 1023-1027. doi: 10.1149/1.2086597

Armand, M. (1983). Polymer solid electrolytes-an overview. Solid State Ion. 9-10, 745-754. doi: 10.1016/0167-2738(83)90083-8

Bates, J. B., Dudney, N. J., Neudecker, B., Ueda, A., and Evans, C. D. (2000). Thin-film lithium and lithium-ion batteries. Solid State Ion. 135, 33-45. doi: 10.1016/S0167-2738(00)00327-1

Bhattacharyya, A. J., and Maier, J. (2004). Second phase effects on the conductivity of non-aqueous salt solutions: "Soggy sand electrolytes." Adv. Mater. 16, 811-814. doi: 10.1002/adma.200306210

Bhattacharyya, R., Key, B., Chen, H., Best, A. S., Hollenkamp, A. F., and Grey, C. P. (2010). In situ NMR observation of the formation of metallic as a good choice. On the other hand, deficiency in strength and toughness of sulfides and polymers calls for toughening or compositing with other solid electrolytes.

Chemical/electrochemical stability are equally important, theoretical calculations, and some experimental results both revealed that the actual stability windows of solid electrolytes are not wide as expected. A stable interface layer with high $\mathrm{Li}^{+}$conductivity and low electrical conductivity is expected. $\mathrm{Li}$ compounds like $\mathrm{LiF}, \mathrm{Li}_{2} \mathrm{~S}, \mathrm{Li}_{2} \mathrm{O}, \mathrm{Li}_{3} \mathrm{~N}$, and $\mathrm{LiNbO}_{3}$ are favorable interface components, while electronic conducting constituents such as metal sulfides (e.g., CoS) and Li-Metal alloys (e.g., Li-Ge alloy) should be avoided. To combine both mechanical and chemical/electrochemical demands of solid electrolyte, developing SPE with high strength and wide electrochemical window is necessary. Compositing is a promising method to utilize synergy effects among various electrolytes, but mitigating the disadvantages of each component need further study. Gel softening interface is also a promising way to achieve intimate contact at electrode-electrolyte interface. But flammable liquid components with narrow electrochemical stability window should be avoided.

According to various requirements, creating an ideal cathodesolid electrolyte interface requires a combination of various factors and methods. By building favorable electrode-electrolyte interface, solid-state batteries with higher safety performance, longer cycle life, and higher energy density are predictable.

\section{AUTHOR CONTRIBUTIONS}

All authors listed have made a substantial, direct and intellectual contribution to the work, and approved it for publication.

\section{FUNDING}

This work was supported by funding from the National Key R\&D Program of China (Grant 2017YFB0102004), National Natural Science Foundation of China (Grant No. 51822211), China Postdoctoral Science Foundation, Beijing Municipal Science \& Technology Commission (Grant Z171100000917021), One Hundred Talent Project of the Chinese Academy of Sciences and Thousand Talents Program for Young Scientists. lithium microstructures in lithium batteries. Nat. Mater. 9, 504-510. doi: $10.1038 /$ nmat 2764

Bruce, P. G. (1995). Structure and electrochemistry of polymer electrolytes. Electrochim. Acta 40, 2077-2085. doi: 10.1016/0013-4686(95) 00144-4

Cao, L., Sun, C., Yuan, Z., Zhang, R., Yuzhuan, S. U., Zhang, S., et al. (2018). Terminology of lithium battery(Draft). Energy Storage Sci. Tech. 7, 148-153.doi: 10.12028/j.issn.2095-4239.2017.0172

Chai, J., Liu, Z., Ma, J., Wang, J., Liu, X., Liu, H., et al. (2017). In situ generation of poly (vinylene carbonate) based solid electrolyte with interfacial stability for $\mathrm{LiCoO}_{2}$ lithium batteries. Adv. Sci. 4:1600377. doi: 10.1002/advs.201600377

Chen, C. H., and Amine, K. (2001). Ionic conductivity, lithium insertion and extraction of lanthanum lithium titanate. Solid State Ionics 144, 51-57. doi: 10.1016/S0167-2738(01)00 884-0 
Chen, L., Li, Y., Li, S. P., Fan, L. Z., Nan, C. W., and Goodenough, J. B. (2018a). $\mathrm{PEO} /$ garnet composite electrolytes for solid-state lithium batteries: from "ceramic-in-polymer" to "polymer-in-ceramic". Nano Energy 46, 176-184. doi: 10.1016/j.nanoen.2017.12.037

Chen, L. Q., Wang, C. Q., Wang, L. Z., Xiao, C. L., and Bi, J. Q. (1980). Lithium ionic conductivity of LISICON single crystals. AcPSn. 29, 661-666.

Chen, R. J., Zhang, Y. B., Liu, T., Xu, B. Q., Lin, Y. H., Nan, C. W., et al. (2017). Addressing the interface issues in all-solid-state bulk-type lithium ion battery via an all-composite approach. ACS Appl. Mater. Interfaces 9, 9654-9661. doi: 10.1021/acsami.6b16304

Chen, S., Wang, J., Zhang, Z., Wu, L., Yao, L., Wei, Z., et al. (2018b). In-situ preparation of poly(ethylene oxide)/ $\mathrm{Li}_{3} \mathrm{PS}_{4}$ hybrid polymer electrolyte with good nanofiller distribution for rechargeable solid-state lithium batteries. J. Power Sour. 387, 72-80. doi: 10.1016/j.jpowsour.2018.03.016

Chien, P. H., Feng, X., Tang, M., Rosenberg, J. T., O’neill, S., Zheng, J., et al. (2018). Li distribution heterogeneity in solid electrolyte $\mathrm{Li}_{10} \mathrm{GeP}_{2} \mathrm{~S}_{12}$ upon electrochemical cycling probed by (7)Li MRI. J. Phys. Chem. Lett. 9, 1990-1998. doi: 10.1021/acs.jpclett.8b00240

Croce, F., Appetecchi, G. B., Persi, L., and Scrosati, B. (1998). Nanocomposite polymer electrolytes for lithium batteries. Nature 394, 456-458. doi: $10.1038 / 28818$

Croce, F., Curini, R., Martinelli, A., Persi, L., Ronci, F., Scrosati, B., et al. (1999). Physical and chemical properties of nanocomposite polymer electrolytes. $J$. Phys. Chem. B 103, 10632-10638. doi: 10.1021/jp992307u

Croce, F., Fiory, F. S., Persi, L., and Scrosati, B. (2001). A high-rate, long-life, lithium nanocomposite polymer electrolyte battery. Electrochem. Solid State Lett. 4, A121-A123. doi: 10.1149/1.1380568

Delmas, C., Nadiri, A., and Soubeyroux, J. L. (1988). The nasicon-type titanium phosphates $\mathrm{LiTi}_{2}\left(\mathrm{PO}_{4}\right)_{3}, \mathrm{NaTi}_{2}\left(\mathrm{PO}_{4}\right)_{3}$ as electrode materials. Solid State Ionics 28, 419-423. doi: 10.1016/S0167-2738(88)80075-4

Deng, F., Wang, X., He, D., Hu, J., Gong, C., Ye, Y. S., et al. (2015). Microporous polymer electrolyte based on PVDF/PEO star polymer blends for lithium ion batteries. J. Membr. Sci. 491, 82-89. doi: 10.1016/j.memsci.2015.05.021

Duan, H., Yin, Y., Guo, Y., and Wan, L. (2017). Research progress on solid-state lithium metal batteries. Energy Storage Sci. Tech. 6, 941-951. doi: 10.12028/j.issn.2095-4239.2017.0087

Fan, L. Z., Dang, Z. M., Shi, Z., and Nan, C. W. (2002). Progress on solid state polymer electrolyte for rechargeable Li battery. Rare Metal. Mat. Eng. 31, 340-344.

Fenton, D. E., Parker, J. M., and Wright, P. V. (1973). Complexes of alkali-metal ions with poly(ethylene oxide). Polymer 14, 589-589. doi: 10.1016/0032-3861(73)90146-8

Fergus, J. W. (2012). Ion transport in sodium ion conducting solid electrolytes. Solid State Ionics 227, 102-112. doi: 10.1016/j.ssi.2012.09.019

Gao, Z., Sun, H., Fu, L., Ye, F., Zhang, Y., Luo, W., et al. (2018). Promises, challenges, and recent progress of inorganic solid-state electrolytes for all-solidstate lithium batteries. Adv. Mater. 30:e1705702. doi: 10.1002/adma.201705702

Glass, A. M., Nassau, K., and Negran, T. J. (1978). Ionic-conductivity of quenched alkali niobate and tantalate glasses. J. Appl. Phys. 49, 4808-4811. doi: $10.1063 / 1.325509$

Gong, Y., Zhang, J., Jiang, L., Shi, J. A., Zhang, Q., Yang, Z., et al. (2017). In situ atomic-scale observation of electrochemical delithiation induced structure evolution of $\mathrm{LiCoO}_{2}$ cathode in a working all-solid-state battery. J. Am. Chem. Soc. 139, 4274-4277. doi: 10.1021/jacs.6b13344

Goodenough, J. B. (2013). Evolution of strategies for modern rechargeable batteries. Acc. Chem. Res. 46, 1053-1061. doi: 10.1021/ar2002705

Han, F., Yue, J., Chen, C., Zhao, N., Fan, X., Ma, Z., et al. (2018). Interphase engineering enabled all-ceramic lithium battery. Joule 2, 497-508. doi: 10.1016/j.joule.2018.02.007

Han, F. D., Zhu, Y. Z., He, X. F., Mo, Y. F., and Wang, C. S. (2016). Electrochemical stability of $\mathrm{Li}_{10} \mathrm{GeP}_{2} \mathrm{~S}_{12}$ and $\mathrm{Li}_{7} \mathrm{La}_{3} \mathrm{Zr}_{2} \mathrm{O}_{12}$ solid electrolytes. Adv. Energy Mater. 6:1501590. doi: 10.1002/aenm.201501590

Han, X., Gong, Y., Fu, K. K., He, X., Hitz, G. T., Dai, J., et al. (2017). Negating interfacial impedance in garnet-based solid-state Li metal batteries. Nat. Mater. 16, 572-579. doi: 10.1038/nmat4821

Han, Y., Wang, Y., Gao, W., Wang, Y., Jiao, L., Yuan, H., et al. (2011). Synthesis of novel CuS with hierarchical structures and its application in lithium-ion batteries. Powder Technol. 212, 64-68. doi: 10.1016/j.powtec.2011.04.028
Haruyama, J., Sodeyama, K., Han, L., Takada, K., and Tateyama, Y. (2014). Space-charge layer effect at interface between oxide cathode and sulfide electrolyte in all-solid-state lithium-ion battery. Chem. Mat. 26, 4248-4255. doi: $10.1021 / \mathrm{cm} 5016959$

Haruyama, J., Sodeyama, K., and Tateyama, Y. (2017). Cation mixing properties toward $\mathrm{Co}$ diffusion at the $\mathrm{LiCoO}_{2}$ cathode/sulfide electrolyte interface in a solid-state battery. ACS Appl. Mater. Interfaces 9, 286-292. doi: 10.1021/acsami.6b08435

Hausbrand, R., Becker, D., and Jaegermann, W. (2014). A surface science approach to cathode/electrolyte interfaces in Li-ion batteries: contact properties, charge transfer and reactions. Prog. Solid State Chem. 42, 175-183. doi: 10.1016/j.progsolidstchem.2014.04.010

$\mathrm{Hu}, \mathrm{C}$., Tao, L., and Yong, Y. (2006). Progress in the study of solid/solid interface between lithium electrode and polymer electrolytes in lithium polymer batteries. Prog. Chem. 18, 542-549. doi: 10.1016/j.polymer.2006.05.069

$\mathrm{Hu}$, E., Wang, X., Yu, X., and Yang, X. Q. (2018). Probing the complexities of structural changes in layered oxide cathode materials for li-ion batteries during fast charge-discharge cycling and heating. Acc. Chem. Res. 51, 290-298. doi: 10.1021/acs.accounts.7b00506

Huang, B., Xu, B., Li, Y., Zhou, W., You, Y., Zhong, S., et al. (2016). Li-ion conduction and stability of perovskite $\mathrm{Li}_{3 / 8} \mathrm{Sr}_{7 / 16} \mathrm{Hf}_{1 / 4} \mathrm{Ta}_{3 / 4} \mathrm{O}_{3}$. ACS Appl. Mater. Interfaces 8, 14552-14557. doi: 10.1021/acsami.6b03070

Huang, B. Y., Wang, Z. X., Chen, L. Q., Xue, R. J., and Wang, F. S. (1996). The mechanism of lithium ion transport in polyacrylonitrilebased polymer electrolytes. Solid State Ionics 91, 279-284. doi: 10.1016/S0167-2738(96)83030-X

Inaguma, Y., Chen, L. Q., Itoh, M., Nakamura, T., Uchida, T., Ikuta, H., et al. (1993). High ionic-conductivity in lithium lanthanum titanate. Solid State Commun. 86, 689-693. doi: 10.1016/0038-1098(93)90841-A

Ito, Y., Yamakawa, S., Hayashi, A., and Tatsumisago, M. (2017). Effects of the microstructure of solid-electrolyte-coated $\mathrm{LiCoO}_{2}$ on its discharge properties in all-solid-state lithium batteries. J. Mater. Chem. A 5, 10658-10668. doi: 10.1039/C7TA01227F

Kato, T., Hamanaka, T., Yamamoto, K., Hirayama, T., Sagane, F., Motoyama, M., et al. (2014). In-situ $\mathrm{Li}_{7} \mathrm{La}_{3} \mathrm{Zr}_{2} \mathrm{O}_{12} / \mathrm{LiCoO}_{2}$ interface modification for advanced all-solid-state battery. J. Power Sour. 260, 292-298. doi: 10.1016/j.jpowsour.2014.02.102

Kato, Y., Hori, S., Saito, T., Suzuki, K., Hirayama, M., Mitsui, A., et al. (2016). Highpower all-solid-state batteries using sulfide superionic conductors. Nat. Energy 1:16030. doi: 10.1038/nenergy.2016.30

Kazyak, E., Chen, K. H., Wood, K. N., Davis, A. L., Thompson, T., Bielinski, A. R., et al. (2017). Atomic layer deposition of the solid electrolyte garnet $\mathrm{Li}_{7} \mathrm{La}_{3} \mathrm{Zr}_{2} \mathrm{O}_{12}$. Chem. Mat. 29, 3785-3792. doi: 10.1021/acs.chemmater.7b00944

Kim, H. S., Oh, Y., Kang, K. H., Kim, J. H., Kim, J., and Yoon, C. S. (2017). Characterization of sputter-deposited $\mathrm{LiCoO}_{2}$ thin film grown on NASICONtype electrolyte for application in all-solid-state rechargeable lithium battery. ACS Appl. Mater. Interfaces 9, 16063-16070. doi: 10.1021/acsami.6b15305

Kim, K. H., Iriyama, Y., Yamamoto, K., Kumazaki, S., Asaka, T., Tanabe, K., et al. (2011). Characterization of the interface between $\mathrm{LiCoO}_{2}$ and $\mathrm{Li}_{7} \mathrm{La}_{3} \mathrm{Zr}_{2} \mathrm{O}_{12}$ in an all-solid-state rechargeable lithium battery. J. Power Sour. 196, 764-767. doi: 10.1016/j.jpowsour.2010.07.073

Kitaura, H., Hayashi, A., Ohtomo, T., Hama, S., and Tatsumisago, M. (2011). Fabrication of electrode-electrolyte interfaces in all-solid-state rechargeable lithium batteries by using a supercooled liquid state of the glassy electrolytes. J. Mater. Chem. 21, 118-124. doi: 10.1039/C0JM01090A

Koerver, R., Aygün, I., Leichtweiß, T., Dietrich, C., Zhang, W., Binder, J. O., et al. (2017). Capacity fade in solid-state batteries: interphase formation and chemomechanical processes in Nickel-rich layered oxide cathodes and lithium thiophosphate solid electrolytes. Chem. Mat. 29, 5574-5582. doi: 10.1021/acs.chemmater.7b00931

Kotobuki, M., Suzuki, Y., Munakata, H., Kanamura, K., Sato, Y., Yamamoto, K., et al. (2011). Effect of sol composition on solid electrode/solid electrolyte interface for all-solid-state lithium ion battery. Electrochim. Acta 56, 1023-1029. doi: 10.1016/j.electacta.2010.11.008

Li, Y., Wang, Z., Cao, Y., Du, F., Chen, C., Cui, Z., et al. (2015). W-doped $\mathrm{Li}_{7} \mathrm{La}_{3} \mathrm{Zr}_{2} \mathrm{O}_{12}$ ceramic electrolytes for solid state $\mathrm{Li}$-ion batteries. Electrochim. Acta 180, 37-42. doi: 10.1016/j.electacta.2015.08.046 
Liang, C. C. (1973). Conduction characteristics of the lithium iodidealuminum oxide solid electrolytes. J. Electrochem. Soc. 120, 1289-1292. doi: $10.1149 / 1.2403248$

Liang, J. Y., Zeng, X. X., Zhang, X. D., Wang, P. F., Ma, J. Y., Yin, Y. X., et al. (2018). Mitigating interfacial potential drop of cathode-solid electrolyte via ionic conductor layer to enhance interface dynamics for solid batteries. J. Am. Chem. Soc. 140, 6767-6770. doi: 10.1021/jacs.8b03319

Lin, D., Liu, W., Liu, Y., Lee, H. R., Hsu, P. C., Liu, K., et al. (2016). High ionic conductivity of composite solid polymer electrolyte via in situ synthesis of monodispersed $\mathrm{SiO}_{2}$ nanospheres in poly(ethylene oxide). Nano Lett. 16, 459-465. doi: 10.1021/acs.nanolett.5b04117

Lin, F., Liu, Y., Yu, X., Cheng, L., Singer, A., Shpyrko, O. G., et al. (2017). Synchrotron X-ray analytical techniques for studying materials electrochemistry in rechargeable batteries. Chem. Rev. 117, 13123-13186. doi: 10.1021/acs.chemrev.7b00007

Liu, N., Li, H., Wang, Z. X., Huang, X. J., and Chen, L. Q. (2006). Origin of solid electrolyte interphase on nanosized $\mathrm{LiCoO}_{2}$. Electrochem. Solid-State Lett. 9, A328-A331. doi: 10.1149/1.2200138

Liu, T., Ren, Y., Shen, Y., Zhao, S. X., Lin, Y., and Nan, C. W. (2016). Achieving high capacity in bulk-type solid-state lithium ion battery based on $\mathrm{Li}_{6.75} \mathrm{La}_{3} \mathrm{Zr}_{1.75} \mathrm{Ta}_{0.25} \mathrm{O}_{12}$ electrolyte: interfacial resistance. J. Power Sour. 324, 349-357. doi: 10.1016/j.jpowsour.2016.05.111

Lu, Y., Li, L., Zhang, Q., Niu, Z., and Chen, J. (2018). Electrolyte and interface engineering for solid-state sodium batteries. Joule 2, 1747-1770. doi: 10.1016/j.joule.2018.07.028

Ma, C., Cheng, Y., Yin, K., Luo, J., Sharafi, A., Sakamoto, J., et al. (2016a). Interfacial stability of $\mathrm{Li}$ metal-solid electrolyte elucidated via in situ electron microscopy. Nano Lett. 16, 7030-7036. doi: 10.1021/acs.nanolett.6b 03223

Ma, J., Liu, Z., Chen, B., Wang, L., Yue, L., Liu, H., et al. (2017). A strategy to make high voltage $\mathrm{LiCoO}_{2}$ compatible with polyethylene oxide electrolyte in all-solid-state lithium ion batteries. J. Electrochem. Soc. 164, A3454-A3461. doi: $10.1149 / 2.0221714$ jes

Ma, Q., Qi, X., Tong, B., Zheng, Y., Feng, W., Nie, J., et al. (2016b). Novel $\mathrm{Li}\left[\left(\mathrm{CF}_{3} \mathrm{SO}_{2}\right)\left(\mathrm{n}-\mathrm{C}_{4} \mathrm{~F}_{9} \mathrm{SO}_{2}\right) \mathrm{N}\right]$-based polymer electrolytes for solid-state lithium batteries with superior electrochemical performance. ACS Appl. Mater. Interfaces 8, 29705-29712. doi: 10.1021/acsami.6b10597

Maier, J. (1995). Ionic-conduction in-space charge regions. Prog. Solid State Chem. 23, 171-263. doi: 10.1016/0079-6786(95)00004-E

Manuel Stephan, A. (2006). Review on gel polymer electrolytes for lithium batteries. Eur. Polym. J. 42, 21-42. doi: 10.1016/j.eurpolymj.2005.09.017

Matsuyama, T., Takano, R., Tadanaga, K., Hayashi, A., and Tatsumisago, M. (2016). Fabrication of all-solid-state lithium secondary batteries with amorphous $\mathrm{TiS}_{4}$ positive electrodes and $\mathrm{Li}_{7} \mathrm{La}_{3} \mathrm{Zr}_{2} \mathrm{O}_{12}$ solid electrolytes. Solid State Ionics 285, 122-125. doi: 10.1016/j.ssi.2015.05.025

Miara, L., Windmüller, A., Tsai, C. L., Richards, W. D., Ma, Q., Uhlenbruck, S., et al. (2016). About the compatibility between high voltage spinel cathode materials and solid oxide electrolytes as a function of temperature. ACS Appl. Mater. Interfaces 8, 26842-26850. doi: 10.1021/acsami.6b09059

Murugan, R., Thangadurai, V., and Weppner, W. (2007). Fast lithium ion conduction in garnet-type $\mathrm{Li}_{7} \mathrm{La}_{3} \mathrm{Zr}_{2} \mathrm{O}_{12}$. Angew. Chem. Int. Ed. Engl. 46, 7778-7781. doi: 10.1002/anie.200701144

Nagao, K., Hayashi, A., Deguchi, M., Tsukasaki, H., Mori, S., and Tatsumisago, M. (2017). Amorphous $\mathrm{LiCoO}_{2}-\mathrm{Li}_{2} \mathrm{SO}_{4}$ active materials: potential positive electrodes for bulk-type all-oxide solid-state lithium batteries with high energy density. J. Power Sour. 348, 1-8. doi: 10.1016/j.jpowsour.2017.02.038

Nakayama, M., Wada, S., Kuroki, S., and Nogami, M. (2010). Factors affecting cyclic durability of all-solid-state lithium polymer batteries using poly(ethylene oxide)-based solid polymer electrolytes. Energy Environ. Sci. 3, 1995-2002. doi: $10.1039 / \mathrm{c} 0 \mathrm{ee} 00266 \mathrm{f}$

Oh, G., Hirayama, M., Kwon, O., Suzuki, K., and Kanno, R. (2016). Bulk-type all solid-state batteries with $5 \mathrm{~V}$ class $\mathrm{LiNi}_{0.5} \mathrm{Mn}_{1.5} \mathrm{O}_{4}$ cathode and $\mathrm{Li}_{10} \mathrm{GeP}_{2} \mathrm{~S}_{12}$ solid electrolyte. Chem. Mat. 28, 2634-2640. doi: 10.1021/acs.chemmater.5b04940

Ohta, N., Takada, K., Zhang, L., Ma, R., Osada, M., and Sasaki, T. (2006). Enhancement of the high-rate capability of solid-state lithium batteries by nanoscale interfacial modification. Adv. Mater. 18, 2226-2229. doi: 10.1002/adma.200502604
Ohta, S., Komagata, S., Seki, J., Saeki, T., Morishita, S., and Asaoka, T. (2013). All-solid-state lithium ion battery using garnet-type oxide and $\mathrm{Li}_{3} \mathrm{BO}_{3}$ solid electrolytes fabricated by screen-printing. J. Power Sour. 238, 53-56. doi: 10.1016/j.jpowsour.2013.02.073

Ohta, S., Seki, J., Yagi, Y., Kihira, Y., Tani, T., and Asaoka, T. (2014). Co-sinterable lithium garnet-type oxide electrolyte with cathode for all-solid-state lithium ion battery. J. Power Sour. 265, 40-44. doi: 10.1016/j.jpowsour.2014.04.065

Okumura, T., Nakatsutsumi, T., Ina, T., Orikasa, Y., Arai, H., Fukutsuka, T., et al. (2011). Depth-resolved X-ray absorption spectroscopic study on nanoscale observation of the electrode-solid electrolyte interface for all solid state lithium ion batteries. J. Mater. Chem. 21, 10051-10060. doi: 10.1039/c0jm04366d

Pal, P., and Ghosh, A. (2018). Influence of $\mathrm{TiO}_{2}$ nano-particles on charge carrier transport and cell performance of $\mathrm{PMMA}^{-\mathrm{LiClO}_{4}}$ based nano-composite electrolytes. Electrochim. Acta 260, 157-167. doi: 10.1016/j.electacta.2017.11.070

Park, C. H., Kim, D. W., Prakash, J., and Sun, Y. K. (2003). Electrochemical stability and conductivity enhancement of composite polymer electrolytes. Solid State Ionics 159, 111-119. doi: 10.1016/S0167-2738(03)00025-0

Park, K., Yu, B. C., Jung, J. W., Li, Y., Zhou, W., Gao, H., et al. (2016). Electrochemical nature of the cathode interface for a solid-state lithium-ion battery: interface between $\mathrm{LiCoO}_{2}$ and garnet- $\mathrm{Li}_{7} \mathrm{La}_{3} \mathrm{Zr}_{2} \mathrm{O}_{12}$. Chem. Mat. 28, 8051-8059. doi: 10.1021/acs.chemmater.6b03870

Porcarelli, L., Gerbaldi, C., Bella, F., and Nair, J. R. (2016). Super soft all-ethylene oxide polymer electrolyte for safe all-solid lithium batteries. Sci Rep. 6:19892. doi: $10.1038 /$ srep 19892

Romanenko, K., Jin, L., Howlett, P., and Forsyth, M. (2016). In situ MRI of operating solid-statelithium metal cells based on ionic plastic crystal electrolytes. Chem. Mat. 28, 2844-2851. doi: 10.1021/acs.chemmater.6b00797

Sakuda, A., Hayashi, A., and Tatsumisago, M. (2013). Sulfide solid electrolyte with favorable mechanical property for all-solid-state lithium battery. Sci Rep. 3:2261. doi: 10.1038/srep02261

Sakuda, A., Kitaura, H., Hayashi, A., Tatsumisago, M., Hosoda, Y., Nagakane, T., et al. (2012). All-solid-state lithium secondary batteries using $\mathrm{Li}_{2} \mathrm{~S}-\mathrm{P}_{2} \mathrm{~S}_{5}$ solid electrolytes and $\mathrm{LiFePO}_{4}$ electrode particles with amorphous surface layer. Chem. Lett. 41, 260-261. doi: 10.1246/cl.2012.260

Seino, Y., Ota, T., and Takada, K. (2011). High rate capabilities of all-solid-state lithium secondary batteries using $\mathrm{Li}_{4} \mathrm{Ti}_{5} \mathrm{O}_{12}$ coated $\mathrm{LiNi}_{0.8} \mathrm{Co}_{0.15} \mathrm{Al}_{0.05} \mathrm{O}_{2}$ and a sulfide-based solid electrolyte. J. Power Sour. 196, 6488-6492. doi: 10.1016/j.jpowsour.2011. 03.090

Shin, B. R., Nam, Y. J., Oh, D. Y., Kim, D. H., Kim, J. W., and Jung, Y. S. (2014). Comparative study of $\mathrm{TiS}_{2} / \mathrm{Li}$-In all-solid-state lithium batteries using glass-ceramic $\mathrm{Li}_{3} \mathrm{PS}_{4}$ and $\mathrm{Li}_{10} \mathrm{GeP}_{2} \mathrm{~S}_{12}$ solid electrolytes. Electrochim. Acta 146, 395-402. doi: 10.1016/j.electacta.2014.08.139

Takamatsu, D., Koyama, Y., Orikasa, Y., Mori, S., Nakatsutsumi, T., Hirano, T., et al. (2012). First in situ observation of the $\mathrm{LiCoO}_{2}$ electrode/electrolyte interface by total-reflection X-ray absorption spectroscopy. Angew. Chem. Int. Ed. Engl. 51, 11597-11601. doi: 10.1002/anie.201203910

Tarascon, J. M., and Armand, M. (2001). Issues and challenges facing rechargeable lithium batteries. Nature 414, 359-367. doi: 10.1038/35104644

Thangadurai, V., Pinzaru, D., Narayanan, S., and Baral, A. K. (2015). Fast solidstate $\mathrm{Li}$ ion conducting garnet-type structure metal oxides for energy storage. J. Phys. Chem. Lett. 6, 292-299. doi: 10.1021/jz501828v

Tong, Y., Chen, L., He, X., and Chen, Y. (2014). Free mesogen assisted assembly of the star-shaped liquid-crystalline copolymer/polyethylene oxide solid electrolytes for lithium ion batteries. Electrochim. Acta 118, 33-40. doi: 10.1016/j.electacta.2013.11.072

Wagner, C. (1972). Electrical conductivity of semiconductors involving inclusions of another phase. J. Phys. Chem. Solids 33, 1051-1052. doi: 10.1016/S0022-3697(72)80265-8

Wakayama, H., Yonekura, H., and Kawai, Y. (2016). Three-dimensional bicontinuous nanocomposite from a self assembled block copolymer for a highcapacity all-solid-state lithium battery cathode. Chem. Mat. 28, 4453-4459. doi: 10.1021/acs.chemmater.6b01665

Wang, A. L., Xu, H., Zhou, Q., Liu, X., Li, Z. Y., Gao, R., et al. (2016a). A new all-solid-state hyperbranched star polymer electrolyte for lithium ion batteries: Synthesis and electrochemical properties. Electrochim. Acta 212, 372-379. doi: $10.1016 /$ j.electacta.2016.07.003 
Wang, C., Gong, Y., Dai, J., Zhang, L., Xie, H., Pastel, G., et al. (2017a). In situ neutron depth profiling of lithium metal-garnet interfaces for solid state batteries. J. Am. Chem. Soc. 139, 14257-14264. doi: 10.1021/jacs.7b07904

Wang, C., Yang, Y., Liu, X., Zhong, H., Xu, H., Xu, Z., et al. (2017b). Suppression of lithium dendrite formation by using LAGP-PEO (LiTFSI) composite solid electrolyte and lithium metal anode modified by PEO (LiTFSI) in allsolid-state lithium batteries. ACS Appl. Mater. Interfaces 9, 13694-13702. doi: 10.1021/acsami.7b00336

Wang, D. W., Zhong, G. M., Dolotko, O., Li, Y. X., Mcdonald, M. J., Mi, J. X., et al. (2014). The synergistic effects of $\mathrm{Al}$ and $\mathrm{Te}$ on the structure and $\mathrm{Li}+$ mobility of garnet-type solid electrolytes. J. Mater. Chem. A 2, 20271-20279. doi: 10.1039/C4TA03591G

Wang, Z., Santhanagopalan, D., Zhang, W., Wang, F., Xin, H. L., He, K., et al. (2016b). In situ STEM-EELS observation of nanoscale interfacial phenomena in all-solid-state batteries. Nano Lett. 16, 3760-3767. doi: 10.1021/acs.nanolett.6b01119

Wenzel, S., Leichtweiss, T., Krüger, D., Sann, J., and Janek, J. (2015). Interphase formation on lithium solid electrolytes-An in situ approach to study interfacial reactions by photoelectron spectroscopy. Solid State Ion. 278, 98-105. doi: 10.1016/j.ssi.2015.06.001

Wenzel, S., Randau, S., Leichtwei, B, T., Weber, D. A., Sann, J., Zeier, W. G., et al. (2016). Direct observation of the interfacial instability of the fast ionic conductor $\mathrm{Li}_{10} \mathrm{GeP}_{2} \mathrm{~S}_{12}$ at the lithium metal anode. Chem. Mat. 28, 2400-2407. doi: 10.1021/acs.chemmater.6b00610

Weston, J. E., and Steele, B. C. H. (1982). Effects of inert fillers on the mechanical and electrochemical properties of lithium salt poly (ethylene-oxide) polymer electrolytes. Solid State Ion. 7, 75-79. doi: 10.1016/0167-2738(82)90072-8

$\mathrm{Xu}, \mathrm{K}$. (2004). Nonaqueous liquid electrolytes for lithium-based rechargeable batteries. Chem. Rev. 104, 4303-4417. doi: 10.1021/cr030203g

Xu, L., Tang, S., Cheng, Y., Wang, K., Liang, J., Liu, C., et al. (2018). Interfaces in solid-state lithium batteries. Joule 2, 1991-2015. doi: 10.1016/j.joule.2018.07.009

Yamamoto, K., Iriyama, Y., Asaka, T., Hirayama, T., Fujita, H., Fisher, C. A., et al. (2010). Dynamic visualization of the electric potential in an all-solidstate rechargeable lithium battery. Angew. Chem. Int. Ed. Engl. 49, 4414-4417. doi: 10.1002/anie.200907319

Yamamoto, K., Iriyama, Y., Asaka, T., Hirayama, T., Fujita, H., Nonaka, K., et al. (2012). Direct observation of lithium-ion movement around an in-situ-formed-negative-electrode/solid-state-electrolyte interface during initial charge-discharge reaction. Electrochem. Commun. 20, 113-116. doi: 10.1016/j.elecom.2012.04.013

Yang, Q., Huang, J., Li, Y., Wang, Y., Qiu, J., Zhang, J., et al. (2018). Surfaceprotected $\mathrm{LiCoO}_{2}$ with ultrathin solid oxide electrolyte film for high-voltage lithium ion batteries and lithium polymer batteries. J. Power Sour. 388, 65-70. doi: 10.1016/j.jpowsour.2018.03.076

Yao, X., Liu, D., Wang, C., Long, P., Peng, G., Hu, Y. S., et al. (2016). Highenergy all-solid-state lithium batteries with ultralong cycle life. Nano Lett. 16, 7148-7154. doi: 10.1021/acs.nanolett.6b03448

Yoon, K., Kim, J. J., Seong, W. M., Lee, M. H., and Kang, K. (2018). Investigation on the interface between $\mathrm{Li}_{10} \mathrm{GeP}_{2} \mathrm{~S}_{12}$ electrolyte and carbon conductive agents in all-solid-state lithium battery. Sci. Rep. 8:8066. doi: 10.1038/s41598-018-26101-4

Yoshima, K., Harada, Y., and Takami, N. (2016). Thin hybrid electrolyte based on garnet-type lithium-ion conductor $\mathrm{Li}_{7} \mathrm{La}_{3} \mathrm{Zr}_{2} \mathrm{O}_{12}$ for $12 \mathrm{~V}$-class bipolar batteries. J. Power Sour. 302, 283-290. doi: 10.1016/j.jpowsour.2015.10.031

Zewde, B. W., Carbone, L., Greenbaum, S., and Hassoun, J. (2018). A novel polymer electrolyte membrane for application in solid state lithium metal battery. Solid State Ion. 317, 97-102. doi: 10.1016/j.ssi.2017.12.039

Zhai, H., Xu, P., Ning, M., Cheng, Q., Mandal, J., and Yang, Y. (2017). A flexible solid composite electrolyte with vertically aligned and connected ion-conducting nanoparticles for lithium batteries. Nano Lett. 17, 3182-3187. doi: 10.1021/acs.nanolett.7b00715
Zhang, D., Yana, H., Zhu, Z., Zhang, H., Wang, J. (2011). Electrochemical stability of lithium bis(oxatlato) borate containing solid polymer electrolyte for lithium ion batteries. J. Power Sour. 196, 10120-10125. doi: 10.1016/j.jpowsour.2011. 07.056

Zhang, J., Zang, X., Wen, H., Dong, T., Chai, J., Li, Y., et al. (2017a). Highvoltage and free-standing poly(propylene carbonate)/ $\mathrm{Li}_{6.75} \mathrm{La}_{3} \mathrm{Zr}_{1.75} \mathrm{Ta}_{0.25} \mathrm{O}_{12}$ composite solid electrolyte for wide temperature range and flexible solid lithium ion battery. J. Mater. Chem. A 5, 4940-4948. doi: 10.1039/C6TA1 0066J

Zhang, J. J., Zhao, J. H., Yue, L. P., Wang, Q. F., Chai, J. C., Liu, Z. H., et al. (2015). Safety-reinforced poly(propylene carbonate)-based all-solid-state polymer electrolyte for ambient-temperature solid polymer lithium batteries. Adv. Energy Mater. 5:1501082. doi: 10.1002/aenm.2015 01082

Zhang, Q., Yao, X., Zhang, H., Zhang, L., and Xiaoxiong, X. U. (2016). Research progress on interfaces of all solid state lithium batteries. Energy Storage Sci. Tech. 5, 659-667. doi: 10.12028/2095-4239.2016.0036

Zhang, W., Richter, F. H., Culver, S. P., Leichtweiss, T., Lozano, J. G., Dietrich, C., et al. (2018). Degradation mechanisms at the $\mathrm{Li}_{10} \mathrm{GeP}_{2} \mathrm{~S}_{12} / \mathrm{LiCoO}_{2}$ cathode interface in an all-solid-state lithium-ion battery. ACS Appl. Mater. Interfaces 10, 22226-22236. doi: 10.1021/acsami.8b05132

Zhang, Z., Hu, L., Wu, H., Weng, W., Koh, M., Redfern, P. C., et al. (2013). Fluorinated electrolytes for $5 \mathrm{~V}$ lithium-ion battery chemistry. Energy Environ. Sci. 6, 1806-1810. doi: 10.1039/c3ee24414h

Zhang, Z. H., Zhao, Y. R., Chen, S. J., Xie, D. J., Yao, X. Y., Cui, P., et al. (2017b). An advanced construction strategy of all-solid-state lithium batteries with excellent interfacial compatibility and ultralong cycle life. J. Mater. Chem. A 5, 16984-16993. doi: 10.1039/C7TA04320A

Zhao, Y., Wu, C., Peng, G., Chen, X., Yao, X., Bai, Y., et al. (2016). A new solid polymer electrolyte incorporating $\mathrm{Li}_{10} \mathrm{GeP}_{2} \mathrm{~S}_{12}$ into a polyethylene oxide matrix for all-solid-state lithium batteries. J. Power Sour. 301, 47-53. doi: 10.1016/j.jpowsour.2015.09.111

Zheng, H., Xiao, D., Li, X., Liu, Y., Wu, Y., Wang, J., et al. (2014). New insight in understanding oxygen reduction and evolution in solid-state lithium oxygen batteries using an in situ environmental scanning electron microscope. Nano Lett. 14, 4245-4249. doi: 10.1021/nl500862u

Zheng, J., Tang, M., and Hu, Y. Y. (2016). Lithium ion pathway within $\mathrm{Li}_{7} \mathrm{La}_{3} \mathrm{Zr}_{2} \mathrm{O}_{12}$-polyethylene oxide composite electrolytes. Angew. Chem. 128, 12726-12730. doi: 10.1002/ange.201607539

Zhou, W., Wang, S., Li, Y., Xin, S., Manthiram, A., and Goodenough, J. B. (2016). Plating a dendrite-free lithium anode with a polymer/ceramic/polymer sandwich electrolyte. J. Am. Chem. Soc. 138, 9385-9388. doi: 10.1021/jacs.6b05341

Zhu, Y., He, X., and Mo, Y. (2016). First principles study on electrochemical and chemical stability of solid electrolyte-electrode interfaces in all-solid-state Li-ion batteries. J. Mater. Chem. A 4, 3253-3266. doi: 10.1039/C5TA08574H

Zugmann, S., Fleischmann, M., Amereller, M., Gschwind, R. M., Wiemhöfer, H. D., and Gores, H. J. (2011). Measurement of transference numbers for lithium ion electrolytes via four different methods, a comparative study. Electrochim. Acta 56, 3926-3933. doi: 10.1016/j.electacta.2011.02.025

Conflict of Interest Statement: The authors declare that the research was conducted in the absence of any commercial or financial relationships that could be construed as a potential conflict of interest.

Copyright (C) 2018 Nie, Hong, Qiu, Li, Yu, Li and Chen. This is an open-access article distributed under the terms of the Creative Commons Attribution License (CC $B Y)$. The use, distribution or reproduction in other forums is permitted, provided the original author(s) and the copyright owner(s) are credited and that the original publication in this journal is cited, in accordance with accepted academic practice. No use, distribution or reproduction is permitted which does not comply with these terms. 